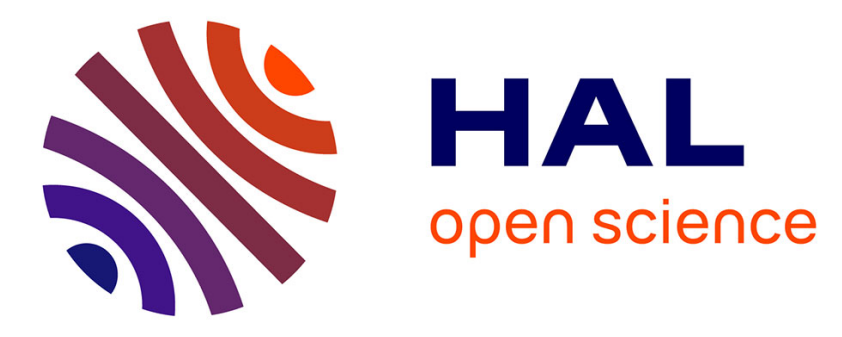

\title{
Experimental parameters for a Cerium 144 based intense electron antineutrino generator experiment at very short baselines
}

J Gaffiot, T Lasserre, G Mention, M Vivier, M Cribier, M Durero, V Fischer, A Letourneau, E Dumonteil, I.S. Saldikov, et al.

\section{To cite this version:}

J Gaffiot, T Lasserre, G Mention, M Vivier, M Cribier, et al.. Experimental parameters for a Cerium 144 based intense electron antineutrino generator experiment at very short baselines. Physical Review D, 2015, 91 (7), pp.072005. 10.1103/PhysRevD.91.072005 . hal-01143757

\author{
HAL Id: hal-01143757 \\ https://hal.science/hal-01143757
}

Submitted on 20 Apr 2015

HAL is a multi-disciplinary open access archive for the deposit and dissemination of scientific research documents, whether they are published or not. The documents may come from teaching and research institutions in France or abroad, or from public or private research centers.
L'archive ouverte pluridisciplinaire HAL, est destinée au dépôt et à la diffusion de documents scientifiques de niveau recherche, publiés ou non, émanant des établissements d'enseignement et de recherche français ou étrangers, des laboratoires publics ou privés. 


\title{
Experimental parameters for a Cerium 144 based intense electron antineutrino generator experiment at very short baselines
}

\author{
J. Gaffiot, ${ }^{1 *}$ T. Lasserre,${ }^{1,2}$ G. Mention, ${ }^{2}$ M. Vivier, ${ }^{2}$ M. Cribier,${ }^{1,2}$ M. Durero, ${ }^{2}$ V. Fischer, ${ }^{2}$ \\ A. Letourneau, ${ }^{2}$ E. Dumonteil, ${ }^{3}$ I. S. Saldikov, ${ }^{4}$ and G. V. Tikhomirov ${ }^{4}$ \\ ${ }^{1}$ Astroparticules et Cosmologie APC, 10 rue Alice Domon et Léonie Duquet, 75205 Paris cedex 13, France \\ ${ }^{2}$ Commissariat a l'énergie atomique et aux énergies alternatives, \\ Centre de Saclay, IRFU, 91191 Gif-sur-Yvette, France \\ ${ }^{3}$ Commissariat a l'énergie atomique et aux énergies alternatives, \\ Centre de Saclay, SERMA, 91191 Gif-sur-Yvette, France \\ ${ }^{4}$ National Research Nuclear University MEPhI (Moscow Engineering Physics Institute), Kashirskoye sh., \\ 31, Moscow 115409, Russia
}

(Received 4 December 2014; published 8 April 2015)

\begin{abstract}
The standard three-neutrino oscillation paradigm, associated with small squared mass splittings $\Delta m^{2} \ll 0.1 \mathrm{eV}^{2}$, has been successfully built up over the last 15 years using solar, atmospheric, long baseline accelerator and reactor neutrino experiments. However, this well-established picture might suffer from anomalous results reported at very short baselines in some of these experiments. If not experimental artifacts, such results could possibly be interpreted as the existence of at least an additional fourth sterile neutrino species, mixing with the known active flavors with an associated mass splitting $\Delta m_{\text {new }}^{2} \gg$ $0.01 \mathrm{eV}^{2}$ and being insensitive to standard weak interactions. Precision measurements at very short baselines $(5-15 \mathrm{~m})$ with intense $\mathrm{MeV} \bar{\nu}_{e}$ emitters can be used to probe these anomalies. In this article, the expected $\bar{\nu}_{e}$ signal and backgrounds of a generic experiment which consists of deploying an intense $\beta^{-}$ radioactive source inside or in the vicinity of a large liquid scintillator detector are studied. The technical challenges to perform such an experiment are identified, along with quantifying the possible source- and detector-induced systematics and their impact on the sensitivity to the observation of neutrino oscillations at short baselines.
\end{abstract}

DOI: 10.1103/PhysRevD.91.072005

PACS numbers: $14.60 . P q$

\section{INTRODUCTION}

Neutrino oscillations have been observed in solar, atmospheric, long baseline accelerator and reactor neutrino experiments [1]. The data collected so far by these experiments are well described in the framework of a three active neutrino mixing approach, in which the three known flavor neutrinos $\left(\nu_{e}, \nu_{\mu}, \nu_{\tau}\right)$ are unitary linear combinations of three mass states $\left(\nu_{1}, \nu_{2}, \nu_{3}\right)$, with squared mass differences of $\Delta m_{21}^{2}=\Delta m_{\mathrm{sol}}^{2}=7.50_{-0.20}^{+0.19} \times 10^{-5} \mathrm{eV}^{2}$ and $\left|\Delta m_{31}^{2}\right| \approx$ $\left|\Delta m_{32}^{2}\right|=\Delta m_{\text {atm }}^{2}=2.32_{-0.08}^{+0.12} \times 10^{-3} \mathrm{eV}^{2} \quad$ [1]. The "sol" and "atm" subscripts historically stand for solar and atmospheric experiments, which provided the first evidence for neutrino oscillations. Beyond this well-established picture, anomalous results have been reported in different neutrino experiments, such as the LSND [2] and MiniBooNE [3,4] accelerator experiments, the calibration of the Gallex and SAGE solar neutrino experiments [5-7] and, more recently, the reactor experiments at short baselines [8]. If not related to experimental issues that are not yet understood, global fits of short-baseline neutrino oscillation experiments show that these anomalies can be interpreted by the addition of one $(3+1)$ or two $(3+2)$

*jonathan.gaffiot@cea.fr sterile neutrinos to the standard three-neutrino paradigm, although no sterile neutrino model currently provides a compelling explanation of all data [8].

This article addresses the possibility and technical feasibility of deploying an intense $\bar{\nu}_{e}$ generator, using a radioactive $\beta^{-}$decaying isotope, outside or inside a large liquid scintillator detector to test these short baseline anomalies. The key experimental parameters of such a radioactive source experiment are identified, and their impact on the sensitivity to short baseline neutrino oscillation is studied. The simplest $(3+1)$ neutrino mixing scheme is considered here, with a fourth massive neutrino state presenting squared mass splittings in the range $\left|\Delta m_{\text {new }}^{2}\right| \sim 0.1-5 \mathrm{eV}^{2}$.

The article is organized as follows: Section II describes the motivations of such an experimental concept, while Sec. III details the choice of the radioactive source to be used as an $\bar{\nu}_{e}$ generator. Section IV reports on sourceinduced backgrounds, activity and $\bar{\nu}_{e}$ energy spectra, which must be accurately characterized in order not to degrade the experimental sensitivity to short baseline oscillations. Section V reviews the existing large liquid scintillator detectors, which meet our experimental requirements. Section VI describes the signature of neutrino oscillations in such large liquid scintillator detectors, as well as the 
impact of source-related and detector-related experimental parameters on the sensitivity to short baseline oscillations. As explained in Sec. IV, minimizing the source-induced backgrounds is of primary importance: Sec. VII therefore presents the results of an accurate simulation of the sourceinduced backgrounds in a large liquid scintillator detector to set the source maximum levels of radioactive impurities. Finally, Sec. VIII is devoted to our conclusions.

\section{EXPERIMENTAL CONCEPT}

An experiment must be sensitive to the deformation of the $\bar{\nu}_{e}$ energy spectrum and/or the deformation of the spatial distribution of $\bar{\nu}_{e}$ interactions to unambiguously test the short baseline oscillation hypothesis. The $\Delta m^{2}$ region coverage is mostly driven by the source-detector distance. Using $\bar{\nu}_{e}$ with $\mathrm{MeV}$ energies, distances of the order of 1 to $10 \mathrm{~m}$ are required to probe the $0.1-5 \mathrm{eV}^{2} \Delta m^{2}$ region preferred by global fits to short baseline neutrino data. A solution meeting this source-detector requirement has already been proposed by placing a "small" liquid scintillator based $\bar{\nu}_{e}$ detector close to a nuclear reactor [9]. In this article we study an alternative solution, consisting in deploying an $\bar{\nu}_{e}$ generator in the vicinity of a liquid scintillator detector, as already proposed in $[10,11]$. Such a solution requires a high-enough source activity and a large-enough detector in order to accumulate a significant statistics over a few mean lifetimes of the $\beta^{-}$decaying radioisotope. Furthermore, a good detector must have the capability of precisely reconstructing both the energy and interaction vertex of each neutrino candidate, in order to observe an oscillation pattern in the $L / E \sim 0.1-10 \mathrm{mMeV}^{-1}$ range. Three suitable detectors, which are (or are soon to be) in operation, have been identified: KamLAND [12], Borexino [13] and SNO+ [14].

These detectors all share features that are important to a clean and unambiguous measurement: They are located deep underground in an ultra-low background environment, they are $\mathcal{O}(10 \mathrm{~m})$-sized spherical detectors filled with ultra-pure hydrogen-rich liquid scintillator, and they have good vertex reconstruction and energy resolution.

A statistics of a few 10000s of $\bar{\nu}_{e}$ interactions, collected over a few emitter's mean lifetimes, is necessary to probe the $\Delta m^{2}$ region preferred by the global fits to short baseline anomalous results. Considering the typical dimensions of the detectors mentioned above, source activities must range from $1 \mathrm{PBq}$ to $10 \mathrm{PBq}$, depending on the source-detector distance and detection volume (fiducial mass of liquid scintillator).

\section{SOURCE RADIOISOTOPE}

The choice of using a $\nu_{e}$ or an $\bar{\nu}_{e}$ source is made upon the consideration of many factors, such as source mean lifetime, source production feasibility, signal detection process and backgrounds coming either from the source or from the detector environment. This section reviews the advantages and drawbacks of such $\nu_{e}$ and $\bar{\nu}_{e}$ sources. A focus on the ${ }^{144} \mathrm{Ce}^{144} \mathrm{Pr}$ pair of radioisotopes, which is in our opinion the best candidate as a radioactive source to be deployed close to a large liquid scintillator detector, is presented.

\section{A. Neutrino sources}

Production of intense $\nu_{e}$ sources and deployment in neutrino detectors have already been performed in the 1990s, for the precise understanding of the Gallex and SAGE [15-17] radiochemical solar neutrino detectors. Such sources emit $\nu_{e}$ through the electron capture process of unstable nuclei. Two nuclei were used at that time, and are still under consideration for a radioactive source experiment at short baselines: ${ }^{51} \mathrm{Cr}$ and ${ }^{37} \mathrm{Ar}$.

${ }^{51} \mathrm{Cr}$ decays with a 27.7 -day half-life and mostly produces $753-\mathrm{keV}$ neutrinos. However, $433-\mathrm{keV}$ neutrinos are produced with a $10 \%$ branching ratio along with a $320-\mathrm{keV}$ gamma. ${ }^{37} \mathrm{Ar}$ produces $814-\mathrm{keV}$ neutrinos with $100 \%$ branching ratio and a 35-day half-life [18]. In terms of heat release, and shielding to gamma rays, ${ }^{37} \mathrm{Ar}$ is easier than ${ }^{51} \mathrm{Cr}$ to handle. It also benefits from a slightly longer half-life and a higher neutrino energy which could help discriminate against gammas from natural radioactivity. Being a metal, ${ }^{51} \mathrm{Cr}$ is chemically easier to extract and manipulate than ${ }^{37} \mathrm{Ar}$.

The realization of such $\nu_{e}$ sources is technically challenging. Both isotopes have to be produced by neutron irradiation inside a nuclear reactor, through ${ }^{50} \mathrm{Cr}(\mathrm{n}, \gamma){ }^{51} \mathrm{Cr}$ and ${ }^{40} \mathrm{Ca}(\mathrm{n}, \alpha){ }^{37} \mathrm{Ar}$ reactions for ${ }^{51} \mathrm{Cr}$ and ${ }^{37} \mathrm{Ar}$, respectively. A very high neutron flux is necessary with multiple irradiation steps to achieve a high-enough specific activity. Furthermore, the $(\mathrm{n}, \alpha)$ reaction has a $1.75-\mathrm{MeV}$ threshold requiring irradiation with fast neutrons to efficiently produce the required level of activity. This is an additional technical challenge to the production of a ${ }^{37} \mathrm{Ar}$ source.

From the transportation point of view, the deployment of ${ }^{a}{ }^{51} \mathrm{Cr}$ or ${ }^{37} \mathrm{Ar}$ source is also challenging. As pointed out above, the half-lives of such radionuclides are of the order of a few tens of days, requiring prompt transportation and deployment logistic solutions to be implemented. This could be a major issue when the suitable nuclear reactor for neutron irradiation and the deployment site are far away, and especially if they are located in two different countries. Moreover, nuclear safety regulations concerning the transportation of radioactive materials are very strict and can, for example, forbid air transportation schemes.

Last but not least, $\nu_{e}$ are detected through inelastic scatterings off electrons, $\nu_{e}+e^{-} \rightarrow \nu_{e}+e^{-}$, having a small cross section relative to the inverse $\beta$ decay process often used to detect $\bar{\nu}_{e}$. Moreover, the detection is very sensitive to backgrounds because the scattering of $\mathcal{O}(1 \mathrm{MeV}) \nu_{e}$ lies in the natural $\beta$ or $\gamma$ ray radioactivity energy range. So far, only the Borexino detector, which 
is designed to study low-energy solar neutrinos, has demonstrated a low-enough background level to efficiently separate a low-rate electron scattering $\nu_{e}$ signal from backgrounds [13]. Finally, an activity of $\sim 500 \mathrm{PBq}$ is necessary to correctly probe the short baseline anomalies.

\section{B. Antineutrino sources}

As opposed to a $\nu_{e}$ source, an $\bar{\nu}_{e}$ source, or antineutrino generator (ANG hereafter), is a $\beta^{-}$decaying nucleus producing $\bar{\nu}_{e}$ over a broad energy spectrum, up to the maximum endpoint energy $\left(\sim Q_{\beta}\right)$ of its available $\beta$ branches. The detection of $\bar{\nu}_{e}$ in liquid scintillator detectors relies on the inverse beta decay (IBD) reaction: $\bar{\nu}_{e}+p \rightarrow e^{+}+n$. The IBD reaction cross section is higher than the cross section of neutrino scattering on electrons by roughly an order of magnitude at $\mathrm{MeV}$ energies. Furthermore, the IBD reaction signature is a time and space coincidence between the positron prompt energy deposition $(\mathrm{d} E / \mathrm{d} x+$ annihilation) and the delayed gamma energy deposition coming from neutron capture. This signature allows a very efficient IBD candidate selection, together with a powerful background rejection. The prompt signal visible energy is $E_{\text {prompt }}^{\mathrm{vis}}=E_{\overline{\nu_{e}}}-M c^{2}+2 m_{e} c^{2}$, where $M=1.293 \mathrm{MeV} / \mathrm{c}^{2}$ is the mass difference between the proton and the neutron and $m_{e}=0.511 \mathrm{MeV} / \mathrm{c}^{2}$ is the electron mass, so that $1.022 \mathrm{MeV}<E_{\text {prompt }}^{\mathrm{vis}}<Q_{\beta}-0.782 \mathrm{MeV}$. In a nondoped scintillator, neutrons are mostly captured on hydrogen atoms, which then release a $2.2-\mathrm{MeV}$ gamma ray. The time distribution between the prompt and delayed events follows an exponential law with a time constant which is equal to the capture time of neutrons on hydrogen, $\tau \sim 200 \mu \mathrm{s}$. Backgrounds of the IBD signal selection are of two types. The first type is the accidental background, which is made from two random energy depositions in a time window roughly corresponding to the hydrogen capture time. For example, such accidental coincidences arise from two gammas coming from the surrounding natural radioactivity and interacting in the target. The second type of background is called correlated background. For instance, spallation of cosmic rays produces fast neutrons that can thermalize and also be captured in the liquid scintillator, faking both a prompt and delayed energy deposition. Fortunately, the above-mentioned backgrounds are negligible in the three detectors under consideration thanks to large overburdens, very low radioactivity materials and thick shieldings from passive materials.

The IBD reaction energy threshold is $1.806 \mathrm{MeV}$, and it requires a source radioisotope with a high endpoint $\beta^{-}$ decay. Since half-life and endpoint energy are strongly anticorrelated quantities for $\beta^{-}$decay, this requirement leads us to look for nuclei with half-lives typically shorter than a day, thus preventing the production and use of an ANG made of a single radioisotope. However, looking for a cascading pair of $\beta^{-}$decaying isotopes, the parent nucleus
TABLE I. Suitable couple for an $\bar{\nu}_{e}$ radioactive source (National Nuclear Data Center, information extracted from the Chart of Nuclides database [18]).

\begin{tabular}{lcc}
\hline \hline Couple & $\tau_{1 / 2}$ of parent & $Q_{\beta^{-}}$of daughter \\
\hline${ }^{42} \mathrm{Ar}-{ }^{42} \mathrm{~K}$ & $33 \mathrm{y}$ & $3.53 \mathrm{MeV}$ \\
${ }^{90} \mathrm{Sr}-{ }^{90} \mathrm{Y}$ & $28.9 \mathrm{y}$ & $2.28 \mathrm{MeV}$ \\
${ }^{106} \mathrm{Ru}^{-106} \mathrm{Rh}$ & $372 \mathrm{~d}$ & $3.55 \mathrm{MeV}$ \\
${ }^{144} \mathrm{Ce}^{144} \mathrm{Pr}$ & $285 \mathrm{~d}$ & $3.00 \mathrm{MeV}$ \\
\hline \hline
\end{tabular}

being a rather long-lived isotope (with month or year-scale half-life) and the daughter nucleus being a short-lived isotope, could circumvent this difficulty. Another requirement is that the daughter isotope must have a $\beta^{-}$endpoint energy as high as possible above the IBD threshold to maximize the IBD reaction rate. Several pairs of isotopes meeting these requirements have been identified by browsing nuclear databases and are displayed in Table I. Among the identified isotopes, the choice of the pair making the best ANG to test the short baseline anomalies is detailed in the next section.

\section{The golden pair: Cerium-praseodymium 144}

The choice of the best couple as a suitable ANG is also driven by production feasibility. ${ }^{90} \mathrm{Sr},{ }^{106} \mathrm{Ru}$ and ${ }^{144} \mathrm{Ce}$ isotopes are fission products found in nuclear reactors and could be extracted from nuclear spent fuel. ${ }^{42} \mathrm{Ar}$ has to be produced through fast neutron irradiation on ${ }^{41} \mathrm{Ar}$, which has a very short half-life $\left(T_{1 / 2}=109 \mathrm{~ms}\right)$. Production of ${ }^{42} \mathrm{Ar}$ then requires a challenging double neutron capture starting from ${ }^{40} \mathrm{Ar}$. It has therefore been rejected as a possible candidate.

Then only ${ }^{90} \mathrm{Sr},{ }^{106} \mathrm{Ru}$ and ${ }^{144} \mathrm{Ce}$ remain as good candidates to make a suitable ANG. They now have to be compared on the basis of their respective production rate in nuclear reactor cores. The cumulative fission yield is the number of nuclei produced per fission when the reactor core is at equilibrium, particularly including decays from short-lived fission products. To a first approximation (long-lived isotopes never reach equilibrium during fuel irradiation), this quantity allows the comparison of the abundance of each couple. It is shown in Table II for thermal fission of ${ }^{235} \mathrm{U}$ and ${ }^{239} \mathrm{Pu}$, which are by far the most abundant fissioned nuclei in current nuclear reactors. ${ }^{106} \mathrm{Ru}$

TABLE II. Cumulative thermal fission yields of ${ }^{106} \mathrm{Ru},{ }^{90} \mathrm{Sr}$ and ${ }^{144} \mathrm{Ce}$ for the two main fissile isotopes fueling nuclear reactors (National Nuclear Data Center, information extracted from the Chart of Nuclides database [18]).

\begin{tabular}{llcc}
\hline \hline & \multicolumn{3}{c}{ Cumulative fission yield (\%) } \\
\cline { 2 - 4 } & \multicolumn{1}{c}{${ }^{106} \mathrm{Ru}$} & ${ }^{90} \mathrm{Sr}$ & ${ }^{144} \mathrm{Ce}$ \\
\hline${ }^{235} \mathrm{U}$ & $0.401(6)$ & $5.78(6)$ & $5.50(4)$ \\
${ }^{239} \mathrm{Pu}$ & $4.35(9)$ & $2.10(4)$ & $3.74(3)$ \\
\hline \hline
\end{tabular}




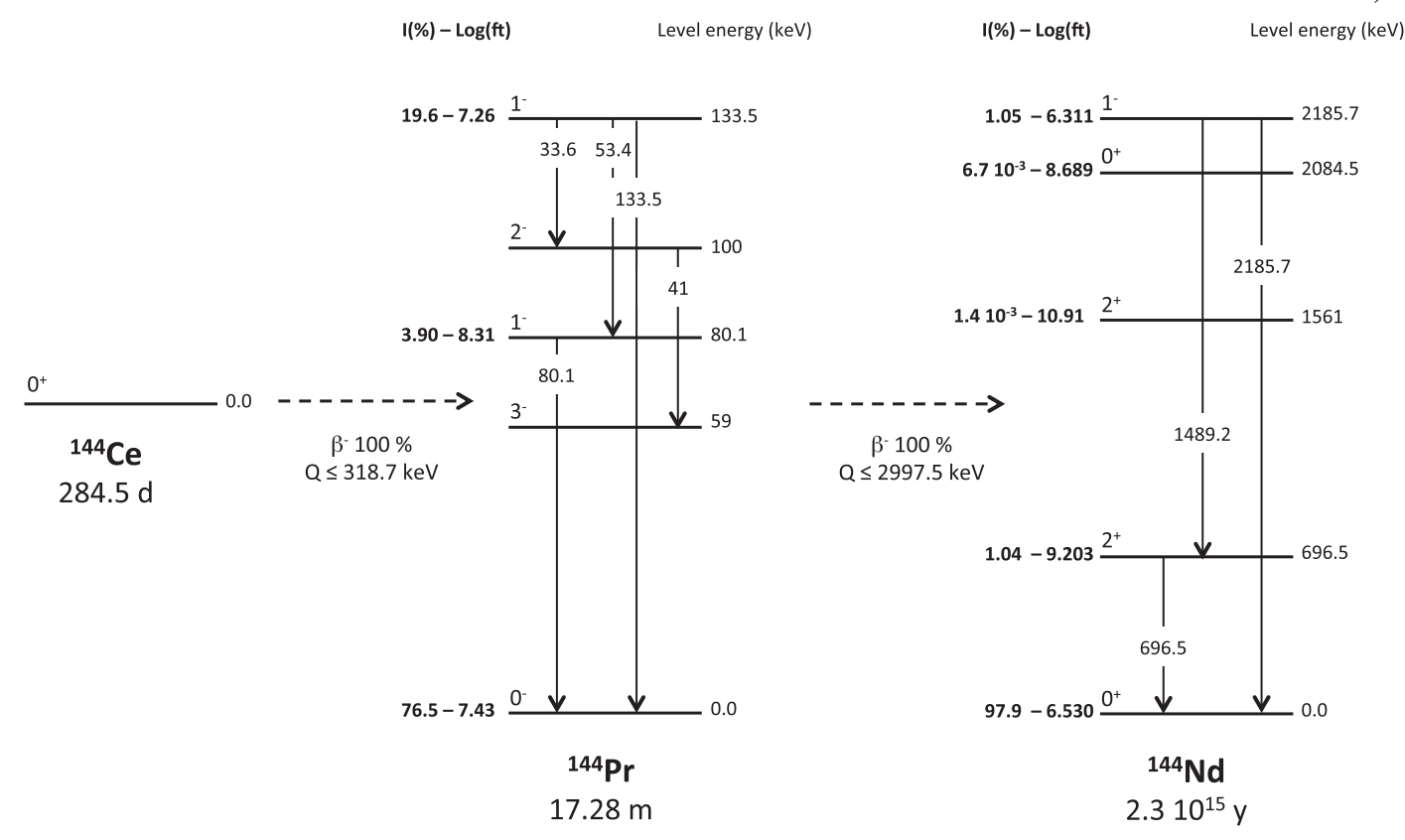

FIG. 1. Simplified decay scheme of the ${ }^{144} \mathrm{Ce}-{ }^{144} \mathrm{Pr}$ couple. $\beta$ branches with branching ratios greater than $0.001 \%$ are displayed, along with the corresponding $\log (\mathrm{ft})$ values, daughter nucleus level energies and spin parities. The main gamma transitions (intensity greater than $0.1 \%$ ) among the excited states of the ${ }^{144} \mathrm{Pr}$ and ${ }^{144} \mathrm{Nd}$ nuclei are also displayed, together with their corresponding energies. The full ${ }^{144} \mathrm{Ce}$ and ${ }^{144} \mathrm{Pr}$ decay scheme data can be found in the National Nuclear Data Center, the Chart of Nuclides database[18].

is disfavored because of its low fission yield for ${ }^{235} \mathrm{U}$, while ${ }^{144} \mathrm{Ce}$ and ${ }^{90} \mathrm{Sr}$ have comparable fission yields for both ${ }^{235} \mathrm{U}$ and ${ }^{239} \mathrm{Pu}$.

Maximizing both the endpoint energy of the daughter nucleus and the source activity (driven by the decay period of the parent nucleus) ultimately leads to rejection of the ${ }^{90} \mathrm{Sr}-{ }^{90} \mathrm{Y}$ couple. Then, ${ }^{144} \mathrm{Ce}-{ }^{144} \mathrm{Pr}$ remains as the best couple for an ANG. Finally, the chemical separation of cerium from other lanthanides is feasible at the industrial scale (see Sec. IVA).

The most suitable ANG for a very short baseline experiment is therefore the ${ }^{144} \mathrm{Ce}-{ }^{144} \mathrm{Pr}$ pair. Depending on the source-detector distance and on the detector target mass, an activity of $2 \mathrm{PBq}$ to $10 \mathrm{PBq}$ (relative to ${ }^{144} \mathrm{Ce} \beta^{-}$ decay rate) is necessary to achieve a statistics of 10000 IBD candidates. This is a factor 10 below the required activities for $\nu_{e}$ sources in the same experimental configuration.

However, ${ }^{144} \operatorname{Pr} \beta^{-}$decay is followed $0.7 \%$ of the time by a $2.185-\mathrm{MeV}$ gamma ray, as can be seen on the ${ }^{144} \mathrm{Ce}^{-144} \mathrm{Pr}$ decay scheme presented in Fig. 1. This gamma ray could fall both in the prompt and delayed energy windows, thus being an additional source of accidental background. Moreover, due to the very high activity of the ANG, this ray constitutes a major radiation protection concern. A dedicated high- $Z$ material shielding, made, for instance, of lead or tungsten, is therefore necessary to suppress this background. As a reference, a 19-cm -thick tungsten alloy shielding with typical density of $18 \mathrm{~g} / \mathrm{cm}^{3}$ provides an attenuation of $3.1 \times 10^{-7}$ to the $2.185 \mathrm{MeV}$ gamma ray, corresponding to a dose at $1 \mathrm{~m}$ of $0.01 \mathrm{mSv} / \mathrm{h}$ for a $5-\mathrm{PBq}$ source. Finally, it is worth noting that the shielding thickness will also be designed to comply with dose limits imposed by any national, institutional or international regulation.

\section{SOURCE CHARACTERIZATION}

Characterizing the ${ }^{144} \mathrm{Ce}^{144} \mathrm{Pr}$ source is a key requirement for achieving a good experimental sensitivity to short baseline neutrino oscillations. First, source composition and level of radioactive impurities have to be accurately controlled and assessed during the production stages to minimize any possible source-induced background. If not controlled to sufficiently low levels, the presence of radioactive impurities in the ${ }^{144} \mathrm{Ce}^{144} \mathrm{Pr}$ source could also bias any activity measurement, especially those using global methods such as calorimetry. Second, source activity and neutrino energy spectrum shape have to be measured to a good accuracy (at the percent level, see Sec. VIC 1) in order to guarantee a good sensitivity, especially in the $\Delta m^{2} \gtrsim 5 \mathrm{eV}^{2}$ region.

\section{A. Composition}

The Russian spent nuclear fuel reprocessing company, Federal State Unitary Enterprise Mayak Production Association (hereafter FSUE "Mayak" PA), has been identified to be the only facility able to deliver a PBq scale sealed source of ${ }^{144} \mathrm{Ce}$. Cerium separation and extraction, together with final source packaging and certification, is an operation 
lasting for several months. The intrinsic abundance of ${ }^{144} \mathrm{Ce}$ along with the extraction process efficiency requires 5 to 10 tons of VVER (from "Vodo-Vodiano Energuetitcheski Reaktor," the Russian pressurized water reactor technology) spent fuel to be reprocessed in order to achieve an activity at the $\mathrm{PBq}$ level. The first step of the cerium extraction is a standard reprocessing of nuclear spent fuel components, leading to a lanthanides and minor actinides concentrate. In a second step, cerium is separated using a complexing displacement chromatography method [see $[19,20]$ and references therein]. No isotopic separation methods are considered in the cerium extraction stage.

The final product will be made of a few kilograms of sintered $\mathrm{CeO}_{2}$ containing a few tens of grams of ${ }^{144} \mathrm{Ce}$ [21]. The other cerium isotopes, ${ }^{140,142} \mathrm{Ce}$, are stable. Inevitably, the extraction process leads to small lanthanide and minor actinide leftovers, that may be penalizing to realize a neutrino experiment. First, these leftovers, if radioactive, could bias the activity measurement. The level of radioactive impurities must therefore be small enough (lower than the desired accuracy, i.e., $\lesssim 1 \%$ ) to account for a negligible contribution to the source activity. This requirement can be achieved using complexing displacement chromatography techniques. Second, the radioactive leftovers can lead to source-induced backgrounds that could degrade the experimental sensitivity to short baseline oscillations. A detailed simulation of source-induced backgrounds in a spherical liquid scintillator detector has been carried out in order to assess the maximum level of such radioactive impurities in the ${ }^{144} \mathrm{Ce}$ source, and it is presented in Sec. VII.

\section{B. Source activity}

As previously stated, a precise knowledge of the source activity is mandatory in order to cover most of the $\Delta m^{2}$ region preferred by the short baseline anomalies. As shown in Sec. VI, a precision at the percent level is necessary and makes any activity measurement very challenging. Direct source activity measurements, either by $\beta$ or $\gamma$ spectroscopic measurement or isotopic measurement of the ${ }^{144} \mathrm{Ce}$ concentration, is complicated by the thick high- $Z$ shielding used to suppress the $2.185-\mathrm{MeV} \gamma$ rays released in the ${ }^{144} \mathrm{Pr}$ decay. Using such methods to precisely estimate the activity then requires source sampling. As such, obtaining a reliable and precise activity measurement further requires that the $\mathrm{CeO}_{2}$ source is homogeneous (i.e., the sample is representative of the ${ }^{144} \mathrm{Ce}^{144} \mathrm{Pr}$ ANG) and that a measurement of a tiny sample mass with a precision below the percent level can be achieved. Regarding the previously mentioned measurement methods, $\beta$ or $\gamma$ spectrometer devices are easier to set up than a mass spectrometer able to handle radioactive materials. Systematic uncertainties associated with such methods are beyond the scope of this article and will be further investigated.
Calorimetric measurements offer an attractive alternative to spectroscopic methods. First, the released heat is directly linked to the source $\beta$-decay activity. Second, it allows the measurement of the source activity without the need for sampling. In such a case, the design of an instrumented calorimeter, dedicated to fitting the source and its associated shielding, is mandatory. Beyond the heat power measurement, great care must be taken in the calculation of the power-to-activity conversion constant. This quantity is calculated using the available information on the different ${ }^{144} \mathrm{Ce}$ and ${ }^{144} \operatorname{Pr} \beta$ branches from nuclear databases, such as branching ratios and $\beta$ mean energies. The uncertainty on those quantities could possibly limit the final activity measurement precision. Using information taken from the Chart of Nuclides [18], the computation of the power-toactivity conversion constant for the ${ }^{144} \mathrm{Ce}-{ }^{144} \mathrm{Pr}$ couple gives $216.0(12) \mathrm{W} / \mathrm{PBq}(0.56 \%$ uncertainty). The $0.56 \%$ uncertainty is dominated here by the uncertainty on the branching ratio of the main ${ }^{144} \operatorname{Pr} \beta$ branch. Furthermore, the $\beta^{-}$model used to compute the mean energy per branch is the LOGFT code [23], which treats any $\beta$ transitions as allowed transitions [23], whereas the main $\beta$ branches of the ${ }^{144} \mathrm{Ce}^{144} \mathrm{Pr}$ couple are nonunique first-forbidden transitions. As explained in the next section, the ${ }^{144} \mathrm{Ce}^{144} \mathrm{Pr}$ energy spectrum shape modeling and $\beta$ spectroscopic measurement will help improve the precision on this conversion constant. The current $\beta$ spectrum shape modeling (see next section) gives $215.6(13) \mathrm{W} / \mathrm{PBq}(0.60 \%$ uncertainty) for the power-to-activity conversion constant. The branching ratio uncertainties are still dominant with a small additional source of uncertainties coming from the corrections applied to Fermi's theory.

\section{Spectral shape: Modeling and $\boldsymbol{\beta}$ spectrometry}

Both the ${ }^{144} \mathrm{Ce}^{1}{ }^{144} \mathrm{Pr}$ source $\beta^{-}$and $\bar{\nu}_{e}$ energy spectra have to be accurately known. As explained in the previous section, calorimetric methods require a very good accuracy on the power-to-activity conversion factor to reach a percent-level precision on the source activity. This conversion factor is related to the mean energy per decay released by the $\beta$ particles and is sensitive to the spectrum shape. A precise knowledge of the full ${ }^{144} \mathrm{Ce}^{-144} \operatorname{Pr} \beta$ spectrum is then necessary to compute this conversion factor to a good precision, which is critical for estimating the ANG activity and then predicting the number of IBD interactions in the detector. Moreover, the number of expected events depends on the fraction of $\bar{\nu}_{e}$ emitted above the IBD threshold. Therefore, the accuracy achieved by a rate + shape analysis (see Sec. VIB) will strongly depend on the $\bar{\nu}_{e}$ spectrum shape uncertainty. To a lesser extent, any uncertainties on the $\bar{\nu}_{e}$ energy spectrum shape will also degrade the experiment free rate sensitivity (see Sec. VIB) to any distortions caused by neutrino oscillations at short baselines. However, this effect is reduced by 

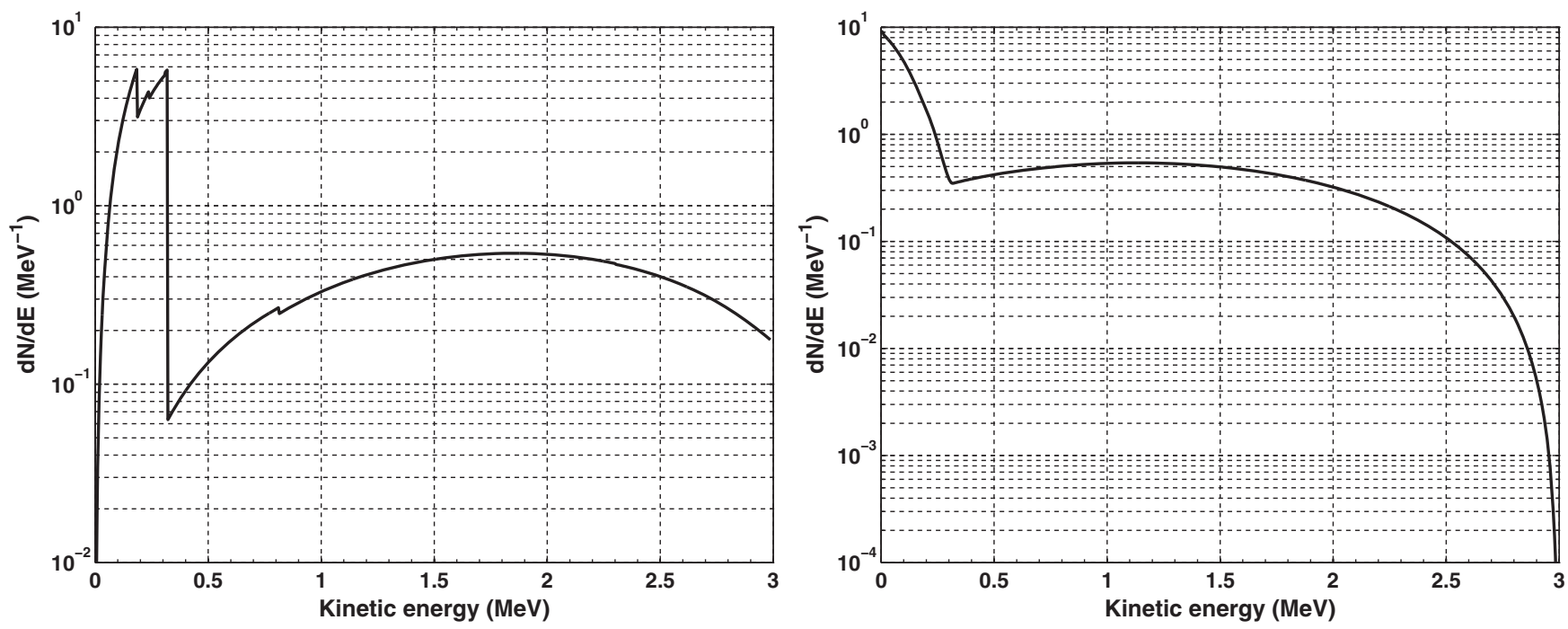

FIG. 2. Estimation of the $\bar{\nu}_{e}$ spectrum without neutrino oscillations (left panel) and of the $\beta$ spectrum (right panel) of the ${ }^{144} \mathrm{Ce}-{ }^{144} \mathrm{Pr}$ couple. Both spectra are normalized to two decays.

the highly characteristic oscillating pattern of the expected signal.

The ${ }^{144} \mathrm{Ce}^{1{ }^{144}} \operatorname{Pr} \beta$ and $\bar{\nu}_{e}$ spectra are a combination of several $\beta$ branches. To a very good approximation, they are linked at the $\beta$ branch level through the following energy conservation relationship $E_{\beta^{-}}=Q_{\beta}-E_{\bar{\nu}_{e}}$, where $E_{\beta^{-}}, Q_{\beta}$ and $E_{\bar{\nu}_{e}}$ are the $\beta$ particle kinetic energy, the $\beta$ branch endpoint energy and the $\bar{\nu}_{e}$ energy, respectively. In addition to the $\beta$ transitions presented in Fig. 1, ${ }^{144} \mathrm{Pr}$ presents seven secondary branches with branching ratios smaller than $0.01 \%$ [18]. Among all possible ${ }^{144} \mathrm{Ce}$ and ${ }^{144} \operatorname{Pr} \beta$ transitions, only two transitions exhibit endpoint energies larger than the IBD reaction energy threshold. They come from the decay of ${ }^{144} \mathrm{Pr}$ and $98.94 \%$ of its decays. Although the other ${ }^{144} \mathrm{Ce}$ and ${ }^{144} \operatorname{Pr} \beta$ branches are irrelevant regarding $\bar{\nu}_{e}$ detection, they have to be carefully studied and measured for the calculation of the power-to-activity conversion factor.

A calculation of the full $\bar{\nu}_{e}$ spectrum, assuming that the ${ }^{144} \mathrm{Ce}-{ }^{144} \mathrm{Pr}$ couple is at secular equilibrium, is shown in Fig. 2. The detected spectrum is shown in Fig. 3. This calculation has been done using Fermi's theory of $\beta$ decay $[24,25]$ of an infinitely massive pointlike nucleus, corrected for various effects such as finite size and mass of the nucleus (considering both electromagnetic and weak interaction), the nucleus recoil effect on the phase space and Coulomb field [26], first-order radiative corrections [27], the screening effect of the atomic electrons [28] and the weak magnetism effect $[25,29]$.

Each effect represents up to a few percent correction to the $\bar{\nu}_{e}$ spectrum shape, except the nucleus recoil effect which has a negligible impact for the ${ }^{144} \mathrm{Ce}$ and ${ }^{144} \mathrm{Pr}$ heavy nuclei. These corrections are generally known with a subpercent precision, except for the weak magnetism effect, for which the few available calculations are uncertain and available data are too sparse to perform a reliable comparison. However, the main ${ }^{144} \operatorname{Pr} \beta$ branch $(97.9 \%$ branching ratio) is not affected by weak magnetism as it is a $0^{-} \rightarrow 0^{+}$nonunique first-forbidden transition with no angular momentum change [30,31]. The $\bar{\nu}_{e}$ spectrum final uncertainty budget is then dominated by the screening correction, which dominates in the high-energy part of the spectrum, and the shape factors of the nonunique firstforbidden $\beta$ branches occurring in the ${ }^{144} \mathrm{Ce}$ and ${ }^{144} \mathrm{Pr}$ decay schemes (see Fig. 1). The final uncertainty budget on the $\bar{\nu}_{e}$ spectrum modeling is of the order of $1 \%$. The impact on the number of expected events has been checked by varying the spectrum shape according to this uncertainty budget. Such a distortion of the spectrum would lead to a

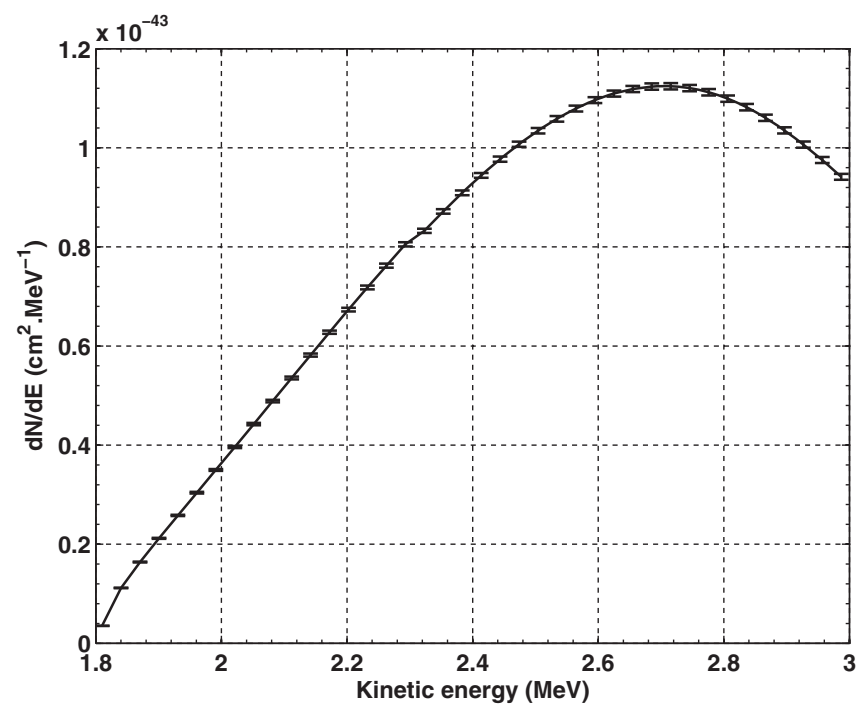

FIG. 3. Differential observable neutrino spectrum from ${ }^{144} \mathrm{Pr}$ folded with the IBD cross section. 
$0.5 \%$ variation in the events number and to a $0.2 \%$ variation

in the power-to-activity conversion factor.

As concerns the modeling of the ${ }^{144} \mathrm{Ce}^{-144} \mathrm{Pr} \beta$ spectrum, the nonunique first-forbidden $\beta$ branch shape factors and corrective terms to Fermi's theory have the same amplitudes and uncertainties as those used to model the $\bar{\nu}_{e}$ spectrum, except the radiative corrections. Radiative corrections are not symmetric between the $\beta$ and $\bar{\nu}_{e}$ spectra. Radiative corrections to the electron spectrum modeling show larger uncertainties and are more difficult to estimate than those corresponding to the $\bar{\nu}_{e}$ spectrum modeling. The radiative correction to the electron spectrum modeling can rise up to a few percent [28].

A precise measurement of the ${ }^{144} \mathrm{Ce}^{1}{ }^{144} \operatorname{Pr} \beta$ spectrum is then necessary to further reduce the shape uncertainties. The $\beta$ spectrum modeling presented previously will be used to interpret the data and possibly to constrain the most uncertain corrections to the first-order Fermi theory. A few challenges arise though for a precise $\beta$ spectroscopic measurement of the ${ }^{144} \mathrm{Ce}-{ }^{144} \mathrm{Pr}$ couple. For example, the short period of ${ }^{144} \mathrm{Pr}$ makes it difficult to measure its associated $\beta$ spectrum independently of the ${ }^{144} \mathrm{Ce} \beta$ spectrum, especially at low energies (i.e., at energies greater than the IBD reaction threshold in the $\bar{\nu}_{e}$ case) where the two spectra overlap. A chemical separation of ${ }^{144} \mathrm{Pr}$ from ${ }^{144} \mathrm{Ce}$ can be performed to circumvent this issue. Because of the short ${ }^{144} \mathrm{Pr}$ half-life, it requires a dedicated separating setup, such as chromatographic columns, installed in the vicinity of the $\beta$ spectrometer.

Several methods are considered to perform the ${ }^{144} \mathrm{Ce}-{ }^{144} \mathrm{Pr} \beta$ spectrometry. They are complementary in the sense that they are sensitive to different instrumental effects. The most precise measurement would be achieved using a magnetic spectrometer. However, such a device could be difficult to find. Semiconductors (silicon or germanium) or plastic scintillator detectors could also be used, provided they are thick enough to stop the most energetic $3 \mathrm{MeV}$ electrons from escaping. The interpretation of these data could be challenging because of backscattering effects at low energies [32]. Finally, liquid scintillator devices can also be used to measure radionuclide $\beta$ spectra. Using such an apparatus would allow the ${ }^{144} \mathrm{Ce}-{ }^{144} \mathrm{Pr}$ mixture to be added to the detection volume, preventing backscattering effects. However, energy resolution would be limited by the scintillator light yield and the light collection efficiency. Moreover, the device's energy response would have to be precisely modeled, especially to correct for the escape of the most energetic $\beta$ particles out of the scintillator volume.

\section{NEUTRINO DETECTORS AND DEPLOYMENT SITES}

Any neutrino detector suitable for the search of meterscaled oscillations should meet the following requirements: kiloton-scale detection volume, target liquid rich in free protons, and low background event rate in the $\mathrm{MeV}$ energy range (consequently located deep underground). They should also be able to lower the energy threshold below $1 \mathrm{MeV}$ (minimum visible energy of an IBD event).

The kiloton scale detection volume is necessary to compensate for the relatively low $\bar{\nu}_{e}$ flux of an ANG with a few $\mathrm{PBq}$ activity. The low-energy threshold requirement prevents the use of current water Cerenkov detectors [33], leaving liquid scintillator based detectors the only suitable candidates. As mentioned in Sec. II, these detectors are, namely, the KamLAND detector located in the Japanese Kamioka Mine [12], the Borexino detector located in the Italian Gran Sasso National Laboratory (LNGS) [13], and the soon to be commissioned $\mathrm{SNO}+$ detector located in the Canadian Sudbury mine laboratory (SNOLAB) [14].

These detectors share a similar layout, based on nested enclosures. Starting from the outer part, the first enclosure is a large cylindrical water tank holding PMTs. It is used as a muon veto and as a first shielding against natural radioactivity and fast neutrons induced by cosmic muons. Going inward, a second spherical vessel is nested within the water tank and is also mounted with a large number of PMTs. The second vessel itself includes a third transparent acrylic vessel (SNO+), or nylon vessel (Borexino, KamLAND), which defines the target volume for the detection of neutrinos. It is filled with hydrogen-rich liquid scintillator. The region between the second and third vessels is filled with a nonscintillating and transparent material, and it is called the buffer region. The buffer region separates the PMTs from the target, ensuring a good optical coupling between the scintillator and PMTs. This design enhances the uniformity of the detector response and further shields the target volume against the radioactivity of the surrounding materials.

The large PMT coverage of the KamLAND, Borexino and $\mathrm{SNO}+$ detectors, associated with their good liquid scintillator properties, allow us to achieve an energy resolution of about $7.5 \%$ at $1 \mathrm{MeV}$ and a vertex resolution better than $15 \mathrm{~cm}$, making them very interesting detectors for performing a short baseline neutrino experiment. The main differences come from their target mass and also the possibility and technical feasibility of deploying a radioactive source in their vicinities. This last point is closely examined in the next paragraphs.

Considering a deployment scenario at KamLAND, the ${ }^{144} \mathrm{Ce}$ ANG could be positioned in the water tank close to the inner detector volume, $10 \mathrm{~m}$ away from the target center. A second solution, technically easier and faster than the previous one, would consist in placing the source in an existing storage room, $12 \mathrm{~m}$ away from the target center. Such a deployment scenario, called CeLAND, has been investigated in detail and is described in Ref. [34]. In a second phase, the ANG could be relocated at the center of the target if hints for a signal show up in the first months of 
data taking. However, such a deployment scenario would require further technical challenges to be solved. For example, it would require increasing the high- $Z$ shielding thickness $(\sim 30-35 \mathrm{~cm})$ with respect to the first phase (see, for example, the preliminary study presented in [11]). It would also need to solve additional technical safety and deployment issues and, more importantly, guarantee a level of gamma and neutron emitting contaminants in the ${ }^{144} \mathrm{Ce}-{ }^{144} \mathrm{Pr}$ source much lower than those previously discussed.

A deployment of a ${ }^{144} \mathrm{Ce}-{ }^{144} \mathrm{Pr}$ ANG next to the main stainless steel vessel of the Borexino detector is actually planned for the end of 2015. Such an experiment is called CeSOX [35]. The ANG would be located in the pit underneath the detector, at a distance of $8.25 \mathrm{~m}$ from the target center. Two possible experimental configurations are worth considering in the deployment of an ANG near Borexino. The first experimental configuration uses the Borexino detector as it is today, i.e., with a fiducial volume defined by a radius of $4.25 \mathrm{~m}$ around the detector center. The second experimental configuration assumes an enlarged detection volume, achieved by doping the buffer oil surrounding the primary target with fluors and wavelength shifters. The detection volume radius would reach $5.5 \mathrm{~m}$, increasing the target mass by a factor 2.2. Finally, the deployment of the ${ }^{144} \mathrm{Ce}^{144} \mathrm{Pr}$ source at the center of the Borexino detector is presently disfavored because of space and mechanical constraints at the detector chimney level. Nevertheless, such a deployment could be realized after the refurbishment of the detector neck in the long term.

A third option concerns the forthcoming $\mathrm{SNO}+$ detector that will start commissioning in the next months. For our sensitivity study, a deployment of the ANG close to the inner detector stainless steel tank, $10 \mathrm{~m}$ away from the target center, will be considered. Among the three detectors being considered, $\mathrm{SNO}+$ appears to be the most suitable for a deployment within the target scintillating volume because of its wide chimney, $1.2 \mathrm{~m}$ in diameter.

The three detectors' main characteristics are detailed in Table III. KamLAND offers the highest density of free protons, which is $25 \%$ higher than in Borexino and $6 \%$ higher than in $\mathrm{SNO}+$. If no fiducial volume cut is applied

TABLE III. The main characteristics of the liquid scintillator detectors suitable to look for short baseline neutrino oscillations.

\begin{tabular}{lcccc}
\hline \hline Detector & $\begin{array}{c}\text { Mass } \\
\text { (ton) }\end{array}$ & Radius $(\mathrm{m})$ & $\begin{array}{c}\text { Density } \\
\left(10^{2} 8 \mathrm{H} / \mathrm{m}^{3}\right)^{\mathrm{a}}\end{array}$ & $\begin{array}{c}\text { \# of H } \\
\left(10^{3} 1\right)^{\mathrm{a}}\end{array}$ \\
\hline KamLAND & 1000 & 6.5 & 6.60 & 7.6 \\
SNO+ & 780 & 6.0 & 6.24 & 5.6 \\
Borexino geo- $\nu$ & $280^{\mathrm{b}}$ & 4.25 & 5.3 & 1.7 \\
Borexino extended & $415^{\mathrm{b}}$ & 5.5 & 5.3 & 3.7 \\
Generic & 600 & 5.5 & 6.24 & 4.3 \\
\hline \hline
\end{tabular}

${ }^{\mathrm{a}} \mathrm{H}$ stands for hydrogen nuclei.

${ }^{\mathrm{b}}$ Data extrapolated from 100 tons in a $3 \mathrm{~m}$ radius. (for example, to suppress source-induced backgrounds), the detection volume available in KamLAND is a factor 4.5 larger than in Borexino and 1.35 larger than in $\mathrm{SNO}+$. In the case of Borexino, this volume difference could be partially compensated by deploying the ${ }^{144} \mathrm{Ce}$ ANG closer to the detector center. As an example, deploying a 3.7 PBq ${ }^{144} \mathrm{Ce}$ ANG at the locations mentioned above for each detector, and for 1.5 years of data taking, leads to $2.9 \times 10^{4}$, $9.2 \times 10^{3}$ and $2.1 \times 10^{4}$ IBD interactions in KamLAND, Borexino $(R=4.25 \mathrm{~m})$, and $\mathrm{SNO}+$, respectively. Increasing the Borexino detection volume such that $R=4.25 \mathrm{~m}$ would give $2.1 \times 10^{4}$ IBD events.

Finally, it is worth mentioning that the detector background conditions will not be identical because of different overburdens and contamination by structure material radioactive impurities. However, the detector-induced backgrounds are expected to be very low compared to the ${ }^{144} \mathrm{Ce}$ ANG signal. If necessary, they can be well identified and measured before or after the ANG deployment. Thus, in the following studies, detector-induced backgrounds will be neglected.

\section{EXPERIMENTAL SENSITIVITY TO SHORT BASELINE OSCILLATIONS}

This section details the expected neutrino signal modeling and sensitivity to short baseline oscillations. The impact of several source and detector related experimental parameters on the sensitivity is also studied.

Unless otherwise stated, the study assumes a generic experimental configuration, where a $3.7 \mathrm{PBq}{ }^{144} \mathrm{Ce}^{144} \mathrm{Pr}$ ANG is deployed $10 \mathrm{~m}$ away from the center of a spherical liquid scintillator detector. The detector is made of an active target of $5.5 \mathrm{~m}$ radius, with a buffer region and muon veto that are both $2 \mathrm{~m}$ thick. The ANG is positioned under the detector below a $10 \mathrm{~cm}$ thick steel plate. It is a cylindrical capsule of $14 \mathrm{~cm}$ radius and height, filled with $\mathrm{CeO}_{2}$, and inserted into a $19 \mathrm{~cm}$ thick tungsten shielding. A sketch of this experimental setup is shown in Fig. 5.

The target liquid scintillator is assumed to be linear alkylbenzene (generally known as LAB), whose density is $0.86 \mathrm{~g} / \mathrm{cm}^{3}$, leading to a target mass of $600 \mathrm{t}$. The detector has a $5 \% / \sqrt{E}$ energy resolution and a $15 \mathrm{~cm}$ vertex resolution. No fiducial volume cut is assumed in the signal and sensitivity predictions. In the no-oscillation scenario $1.610^{4}$ IBD interactions would be expected, with data taking time of 1.5 years. Expected ANG-induced backgrounds are discussed in Sec. VII and summarized in Table VI.

\section{A. Expected signal}

The expected number of $\bar{\nu}_{e}$ events, $N_{\bar{\nu}_{e}}$, in a volume element $\mathrm{d}^{3} \mathcal{V}_{\text {det }}$ located at a distance $\mathrm{L}$ from a pointlike ANG of initial activity $\mathcal{A}_{0}$, during a time $\mathrm{d} t$ and in an energy interval $\mathrm{d} E$, can be expressed as follows: 

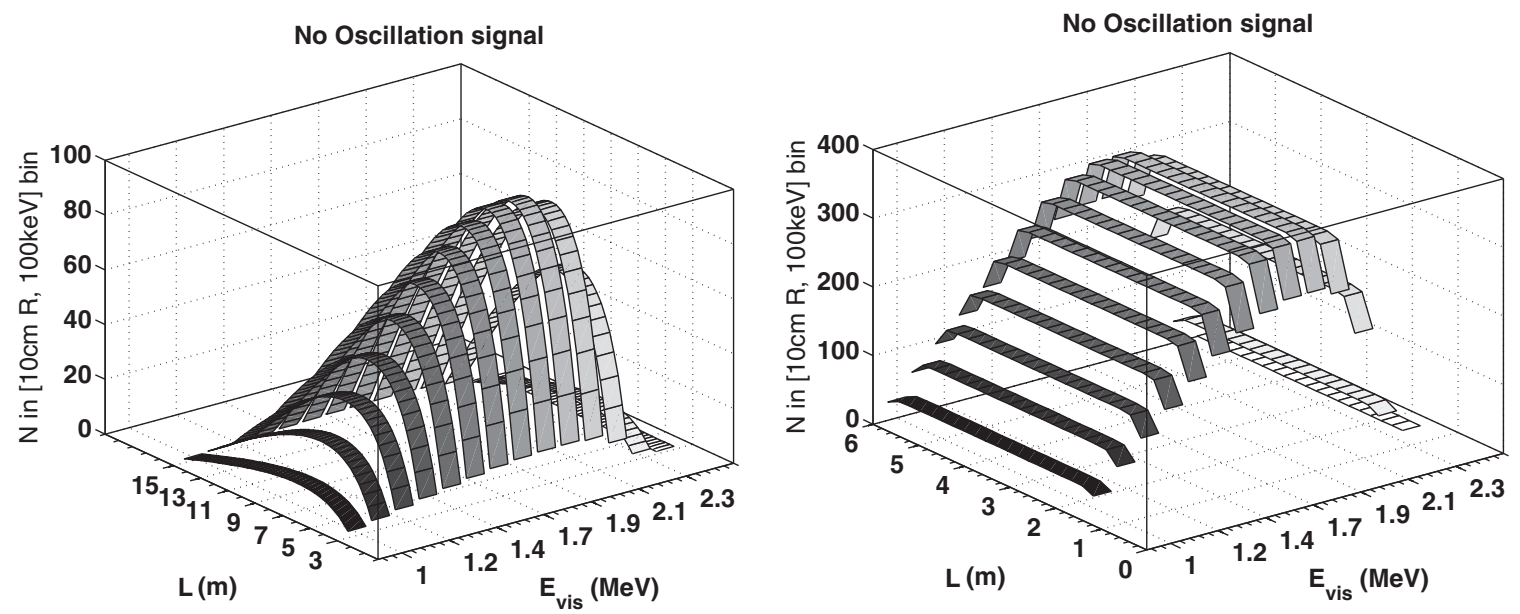

FIG. 4. Expected signal as a function of both visible energy $\mathrm{E}_{\mathrm{vis}}$ and baseline $\mathrm{L}$, in the no-oscillation hypothesis, for an ANG positioned either outside (left) or at the center (right) of a liquid scintillator spherical detector. See text for further details.

$$
\begin{aligned}
\frac{\mathrm{d}^{5} N_{\bar{\nu}_{e}}}{\mathrm{~d} t \mathrm{~d} E \mathrm{~d}^{3} \mathcal{V}_{\mathrm{det}}}= & \mathcal{A}_{0} e^{-t \lambda_{\mathrm{Ce}}} \eta_{p} \varepsilon \frac{1}{4 \pi L^{2}} \sigma_{\mathrm{IBD}}(E) S_{\mathrm{Ce}}(E) \\
& \times \mathcal{P}(L, E),
\end{aligned}
$$

where $\lambda_{\mathrm{Ce}}$ is the ${ }^{144} \mathrm{Ce}$ decay constant $\left(\mathrm{s}^{-1}\right), \eta_{p}$ is the free proton density and $\varepsilon$ is the detection efficiency, which can be position dependent. $\sigma_{\mathrm{IBD}}$ stands for the IBD reaction cross section, and $S_{\mathrm{Ce}}$ is the ${ }^{144} \mathrm{Ce}-{ }^{144} \mathrm{Pr} \bar{\nu}_{e}$ spectrum as computed with the modeling discussed in Sec. IV C. The interpretation of the short baseline anomalies in terms of neutrino oscillation prefers new mass splittings $\Delta m_{\text {new }}^{2} \sim$ $1 \mathrm{eV}^{2} \gg \Delta m_{\mathrm{sol}}^{2}, \Delta m_{\mathrm{atm}}^{2}$. The $\mathcal{P}(L, E)$ survival probability of $\bar{\nu}_{e}$ can then be written assuming a 2-flavor oscillation scenario:

$$
\mathcal{P}(L, E)=1-\sin ^{2}\left(2 \theta_{\text {new }}\right) \sin ^{2}\left(\frac{c^{3}}{\hbar} \frac{\Delta m_{\text {new }}^{2} L}{2 E}\right),
$$

where $\theta_{\text {new }}$ is the new mixing angle associated with the $\Delta m_{\text {new }}^{2}$ new mass splitting.

If the ANG is deployed at the center of the detector, Eq. (1) reduces to

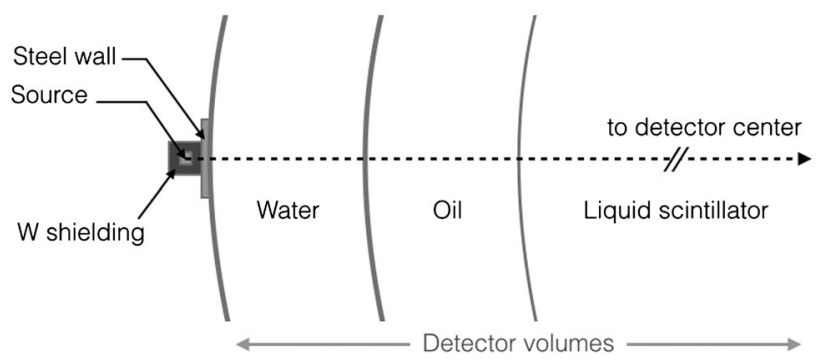

FIG. 5. Sketch of the generic experimental setup.

$$
\frac{\mathrm{d}^{3} N_{\bar{\nu}_{e}}}{\mathrm{~d} t \mathrm{~d} E \mathrm{~d} r}=\mathcal{A}_{0} e^{-t \lambda_{\mathrm{Ce}}} \eta_{p} \varepsilon \sigma_{\mathrm{IBD}}(E) S_{\mathrm{Ce}}(E) \mathcal{P}(r, E),
$$

where the integral over the zenithal and azimuthal coordinates of the detector volume element $\mathrm{d}^{3} \mathcal{V}_{\text {det }}$ (expressed in polar coordinates) has been performed. Equation (3) translates the fact that in the no-oscillation hypothesis, the count rate would be constant as a function of the distance to the center of the detector $r$.

The detector vertex and energy resolution must be taken into account when computing binned spectra. The computation of such spectra is done by integrating Eq. (1) or (3), smeared with Gaussian vertex and energy resolution functions. The expected signal in the absence of oscillations, binned both as a function of $\mathrm{L}$ and $\mathrm{E}$, is shown in Fig. 4 for the generic experimental configuration described above. The $\mathrm{L}$ and $\mathrm{E}$ binned spectrum corresponding to a source positioned at the detector center is also shown for comparison. Because the survival oscillation probability depends only on the L/E ratio, the expected signal as a function of $\mathrm{L} / \mathrm{E}$ has also been calculated and is shown in Fig. 6, for different oscillation scenarios, along with the corresponding "oscillated over non-oscillated" ratios. Computing such a ratio would lead to the $\bar{\nu}_{e}$ survival probability [Eq. (2)] for an ideal detector with no vertex or energy resolutions. The lower panel of Fig. 6 shows that the corresponding oscillations are damped with respect to the raw survival probability because of the detector vertex and energy resolutions. Damping of the oscillation pattern is especially visible at high $\Delta m_{\text {new }}^{2}$ where the oscillation wiggles grow smaller with higher $\mathrm{L} / \mathrm{E}$.

\section{B. Sensitivity and discovery potential}

The sensitivity to short baseline oscillations is evaluated by comparing the observed event rate, binned as a function of both energy and distance, with respect to the expected 


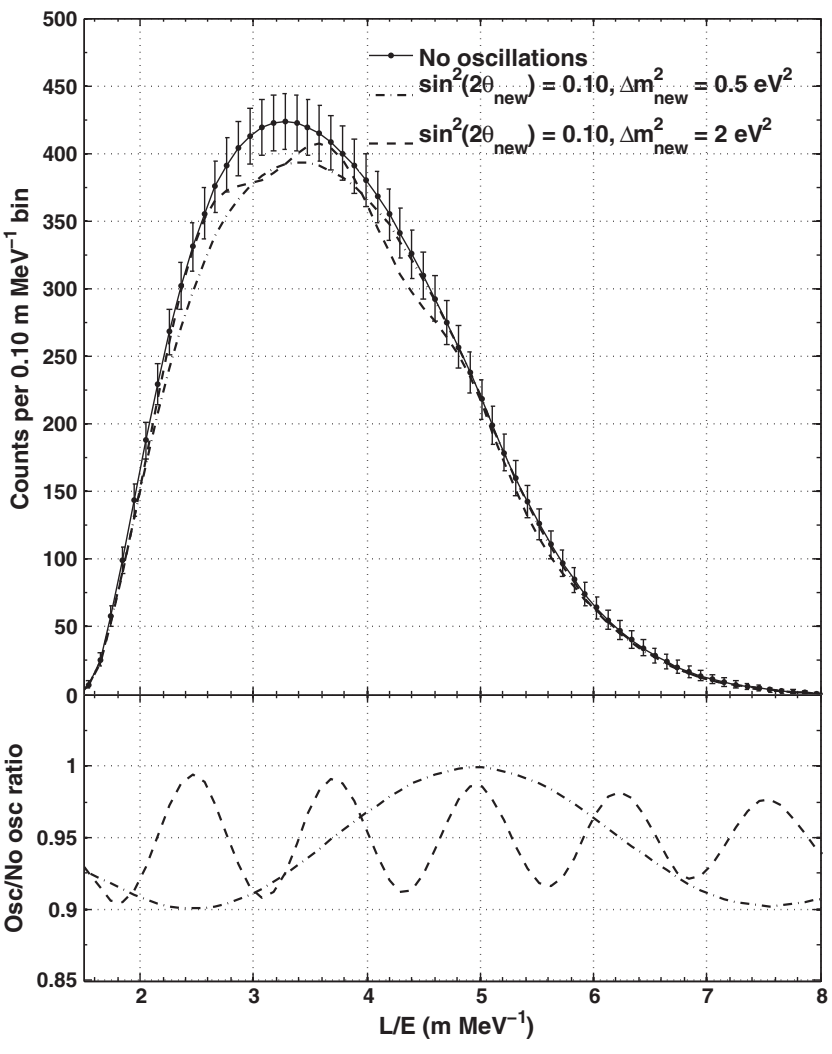

FIG. 6. The upper panel shows the expected signal as a function of L/E. Error bars include statistical uncertainties corresponding to 1.5 years of data taking and a $1.5 \%$ uncertainty on the source activity. The no-oscillation scenario is compared to two oscillation scenarios, with squared mass splittings $\Delta m_{\text {new }}^{2}=0.5$ and $2.0 \mathrm{eV}^{2}$ and $\mathrm{a} \sin ^{2}\left(2 \theta_{\text {new }}\right)=0.1$ mixing parameter. The lower panel shows the ratio of the oscillated spectra to the nonoscillated spectrum.

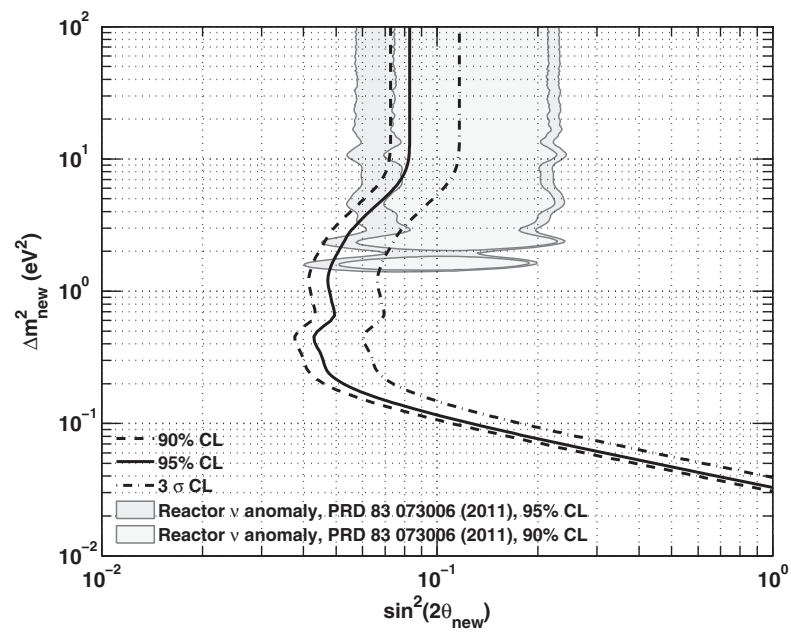

distribution in the presence of oscillations. The following $\chi^{2}$ function is used to test the hypothesis of no oscillation and for $\left(\Delta m_{\text {new }}^{2}, \theta_{\text {new }}\right)$ parameter estimation:

$$
\chi^{2}=\sum_{i, j} \frac{\left(N_{i, j}^{\mathrm{obs}}-(1+\alpha) N_{i, j}^{\mathrm{exp}}\right)^{2}}{N_{i, j}^{\mathrm{exp}}}+\left(\frac{\alpha}{\sigma_{\mathrm{N}}}\right)^{2},
$$

where $N_{i, j}^{\mathrm{obs}}$ and $N_{i, j}^{\mathrm{exp}}$ are the observed and expected number of IBD events in the $i$ th energy bin and $j$ th distance bin, respectively. As explained in the previous section, they are computed according to Eq. (1), taking into account the detector energy and vertex resolution. The nuisance parameter $\alpha$ allows the signal normalization to vary within its associated uncertainty $\sigma_{\mathrm{N}}$. Here, the normalization uncertainty is assumed to come from the uncertainty on the source initial activity $\mathcal{A}_{0}$. Setting the source activity uncertainty $\sigma_{\mathrm{N}}$ to $\infty$ allows an overall floating normalization and a sensitivity study which mostly uses shape distortions to look for oscillations. This is the so-called "free rate" analysis. Setting $\sigma_{\mathrm{N}}$ to the precision achieved by any activity measurement performed prior to the final experiment allows us to use rate information in addition to the information brought by a spectrum shape deformation. This is the so-called "rate + shape" analysis.

After minimizing the $\chi^{2}$ function over the nuisance parameter $\alpha$, the $90 \%, 95 \%$, and $99.73 \%$ exclusion contours are computed as a function of $\Delta m_{\text {new }}^{2}$ and $\theta_{\text {new }}$ such that $\Delta \chi^{2}=\chi^{2}\left(\theta_{\text {new }}, \Delta m_{\text {new }}^{2}\right)-\chi_{\text {min }}^{2}<4.6, \quad 6.0$ and 11.8, respectively. Such contours are shown in Fig. 7 for both rate + shape and free rate analysis, assuming the generic experimental configuration described above.

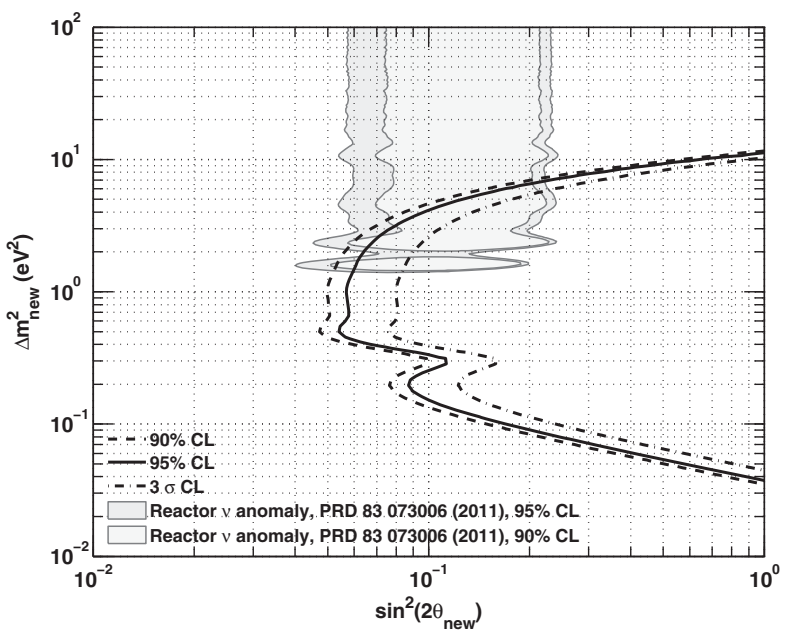

FIG. 7. Sensitivity of our generic reference experiment at 90\%, 95\%, and $99.73 \%$ C.L. The inclusion contours of the reactor antineutrino anomaly are shown in the grey shaded areas. Left panel: Rate + shape sensitivity. Right panel: Free rate sensitivity. Unless explicitly mentioned, default parameters for sensitivity contours include a ${ }^{144} \mathrm{Ce}-{ }^{144} \mathrm{Pr}$ source of $3.7 \mathrm{PBq}$ deployed at $10 \mathrm{~m}$ from the detector center for 1.5 years, with $1.5 \%$ uncertainty on normalization, vertex and energy resolution at $1 \mathrm{MeV}$ of $15 \%$ and $5 \%$, respectively, and contours given at the $95 \%$ C.L. 

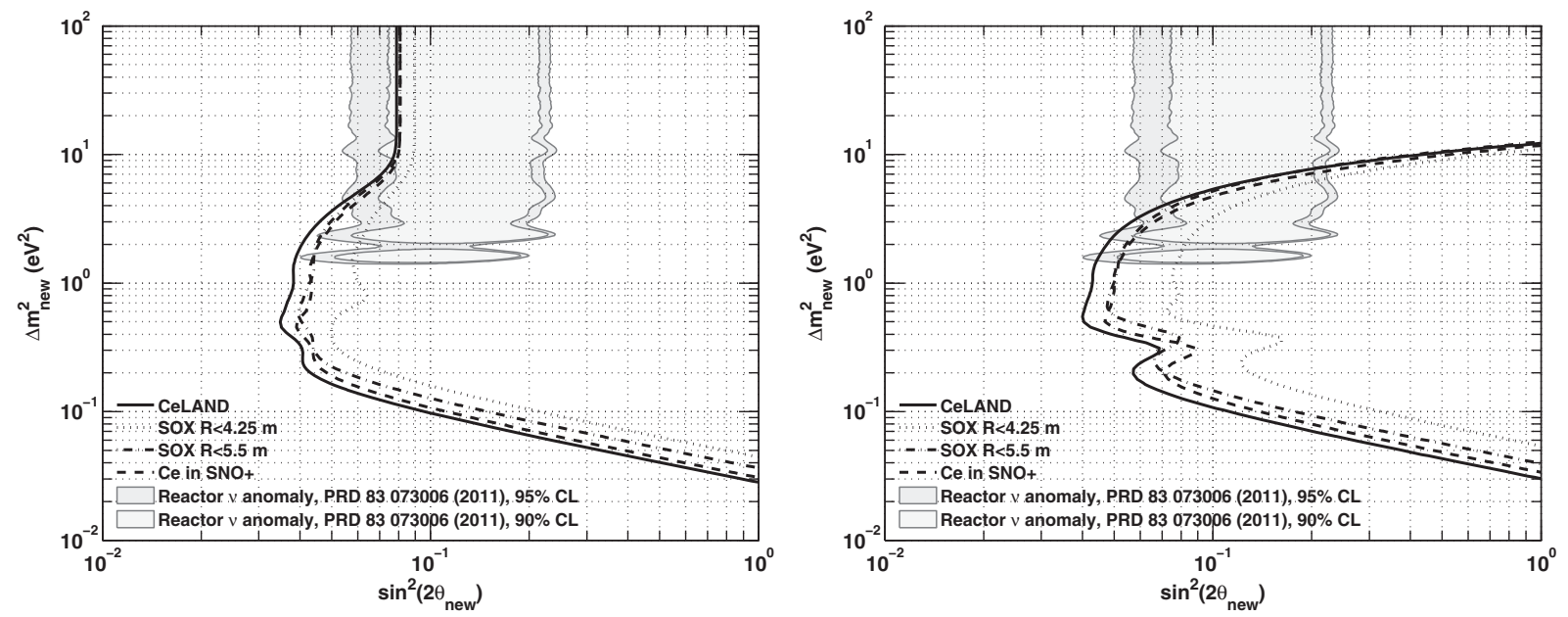

FIG. 8. Sensitivity contours of a source experiment at the KamLAND, Borexino, and SNO+ detectors. See Fig. 7 for parameters. Left panel: Rate + shape sensitivity. Right panel: Free rate sensitivity.

The shape of the sensitivity contours can be understood in the following way. At low $\Delta m_{\text {new }}^{2} \lesssim 0.1 \mathrm{eV}^{2}$, the typical oscillation lengths are much larger than the detector size, and the $\bar{\nu}_{e}$ survival probability approximates to $\mathcal{P}(\mathrm{L}, \mathrm{E}) \approx 1-A \sin ^{2}\left(2 \theta_{\text {new }}\right) \times\left(\Delta m_{\text {new }}^{2}\right)^{2}$, where $\mathrm{A}$ is a constant. In this regime, sensitivity contours defined by constant $\Delta \chi^{2}$ values then impose a linear relationship in logarithmic scale between $\sin ^{2}\left(2 \theta_{\text {new }}\right)$ and $\Delta m_{\text {new }}^{2}$.

In the $\Delta m_{\text {new }}^{2} \sim 0.1-5 \mathrm{eV}^{2}$ regime, oscillation periods are smaller than the detector size but are also small enough so that they are slightly damped by the detector energy and vertex resolutions (see Fig. 6 and related discussion). Moreover, the energy and distance binning sizes, which are of the order of the detector energy and vertex resolutions, are small enough so that the oscillation period is

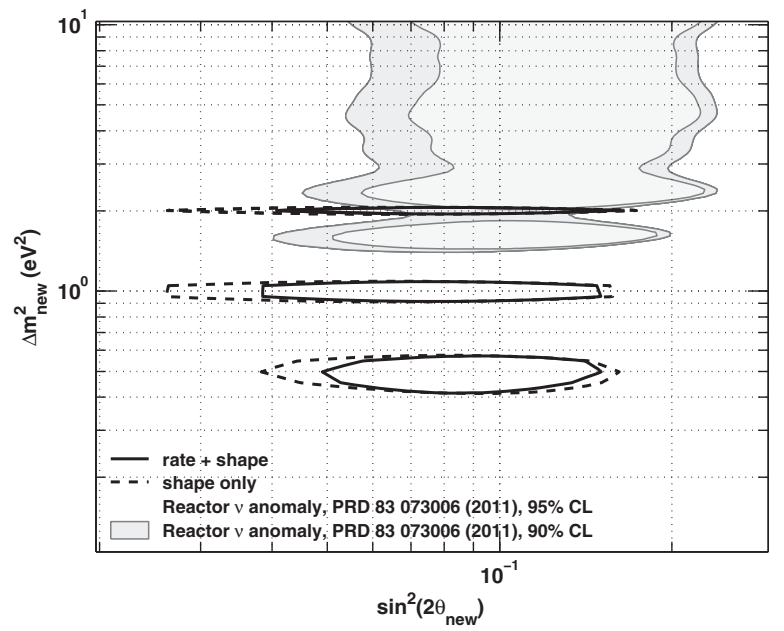

FIG. 9. Illustrative contours for input oscillation signals at $\Delta m_{\text {new }}^{2}=0.5,1,2 \mathrm{eV}^{2}$ and $\sin ^{2}\left(2 \theta_{\text {new }}\right)=0.1$. Acceptance contours are given at the $99 \%$ confidence level. See Fig. 7 for other parameters. properly sampled. This $\Delta m_{\text {new }}^{2}$ regime is the region where the sensitivity to short baseline oscillations is maximum.

Finally, in the high $\Delta m_{\text {new }}^{2} \gtrsim 10 \mathrm{eV}^{2}$ regime, the typical oscillation periods are much smaller than the energy and distance binning sizes and are seriously damped by the detector energy and vertex resolutions. Therefore, the survival probability is averaged out to a constant value, which depends only on the $\sin ^{2}\left(2 \theta_{\text {new }}\right)$ parameter. In a free rate analysis, no information on the $\left(\Delta m_{\text {new }}^{2}, \theta_{\text {new }}\right)$ oscillation parameters can be recovered. In a rate + shape analysis, the rate deficit can be used to infer the $\sin ^{2}\left(2 \theta_{\text {new }}\right)$ mixing parameter, leading to contours which do not depend on the squared mass splitting $\Delta m_{\text {new }}^{2}$.

The estimated sensitivities to short baseline oscillations assuming the deployment scenarios discussed for the KamLAND, Borexino and SNO+ detectors in Sec. V are shown in Fig. 8 for both the rate + shape and free rate analyses. A $1.5 \%$ systematic uncertainty on the initial source activity has been assumed for the rate + shape contours calculation. As expected, the deployment scenario at KamLAND offers the best sensitivity contours among all the detectors discussed previously.

Examples of the discovery potential of a ${ }^{144} \mathrm{Ce}^{144} \mathrm{Pr}$ ANG experiment to short baseline neutrino oscillations, still assuming the generic experimental configuration described previously, are illustrated in Fig. 9. It shows the acceptance contours at the $99 \%$ confidence level of the inferred oscillation parameters if one assumes $\Delta m_{\text {new }}^{2}=0.5,1,2 \mathrm{eV}^{2}$, respectively.

\section{Impact of experimental parameters}

\section{Activity and associated uncertainty}

Figure 10 shows how the source activity affects the sensitivity to short baseline oscillations. Increasing the source activity has little effect in the low $\Delta m_{\text {new }}^{2} \lesssim 0.1 \mathrm{eV}^{2}$ 

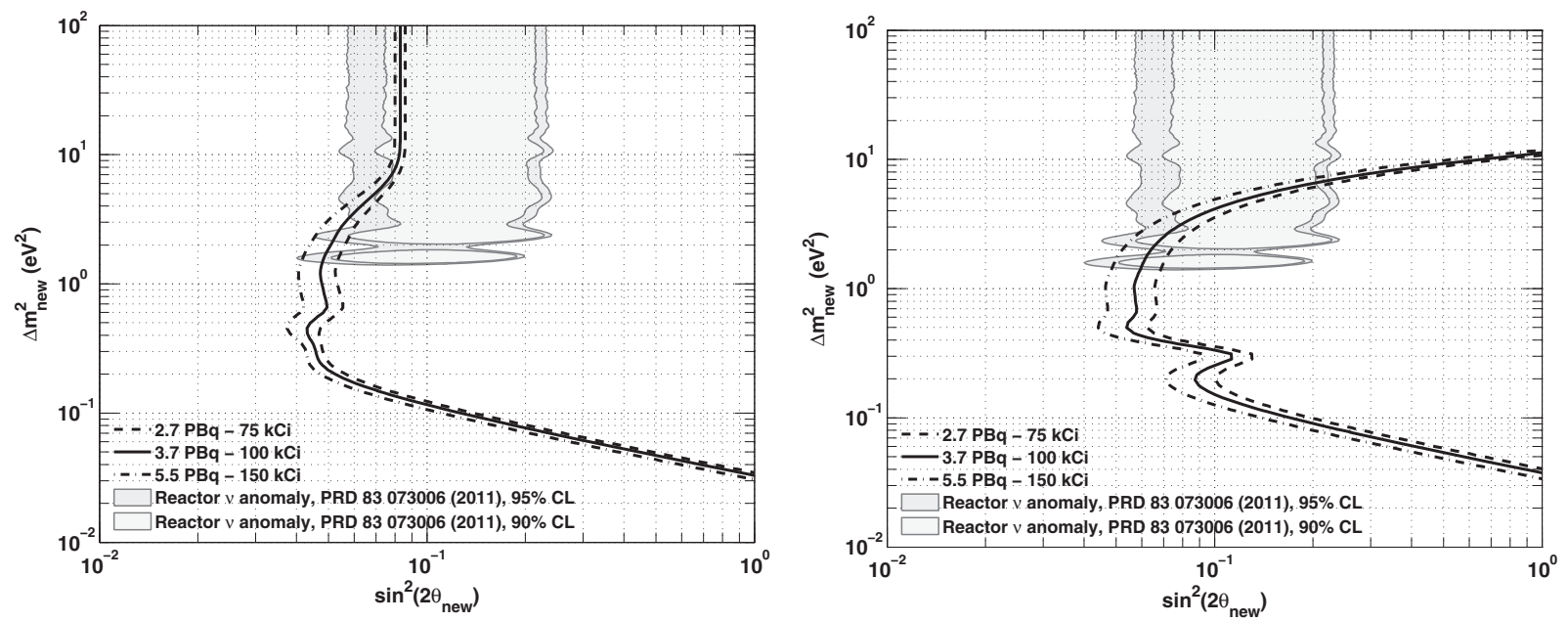

FIG. 10. Influence of source activity on sensitivity, with a rate + shape analysis (left panel) and a free rate analysis (right panel). See Fig. 7 for other parameters.

regime. In this region the oscillation length is larger than the detector size. In this domain of the parameter space, the $\chi^{2}$ sensitivity study compares an overall weighted number of IBD events between the observed and expected signals. Therefore, because of the $\sigma_{\mathrm{N}}$ limiting systematic uncertainty, the sensitivity contours only slightly change with the different source activities studied here. However, the intermediate $0.1 \mathrm{eV}^{2} \lesssim \Delta m_{\text {new }}^{2} \lesssim 10 \mathrm{eV}^{2}$ region is more impacted because in this regime, many oscillation lengths can be sampled in the detector. Increasing the statistics improves the precisions to bin-to-bin changes and thus the search for oscillation patterns. Finally, in the high $\Delta m_{\text {new }}^{2} \gtrsim$ $10 \mathrm{eV}^{2}$ regime, the source activity slightly affects the experimental sensitivity with a rate + shape analysis (Fig. 10, left panel), the free rate analysis being insensitive in that domain (Fig. 10, right panel). The sensitivity is limited here by the $1.5 \%$ normalization uncertainty, which is larger than the $\sim 1 \%$ statistical uncertainty.

The left panel of Fig. 11 illustrates the impact of the source activity knowledge, $\sigma_{\mathrm{N}}$, to the $\chi^{2}$ sensitivity contours. As previously stated, uncertainties on the source activity dominate over statistical uncertainties in the high $\Delta m_{\text {new }}^{2} \gtrsim 10 \mathrm{eV}^{2}$ regime. Degrading the activity uncertainties from $1 \%$ down to $3 \%$ therefore has a strong impact on the sensitivity contours in this region. The lowest part of the sensitivity contours $\left(\Delta m_{\text {new }}^{2} \lesssim 0.5 \mathrm{eV}^{2}\right)$ is also affected because of the measurement degeneracy between $\Delta m_{\text {new }}^{2}$ and $\sin ^{2}\left(2 \theta_{\text {new }}\right)$ in this region where the sensitivity only depends on an overall weighting of events. A better knowledge of the source's activity improves the global
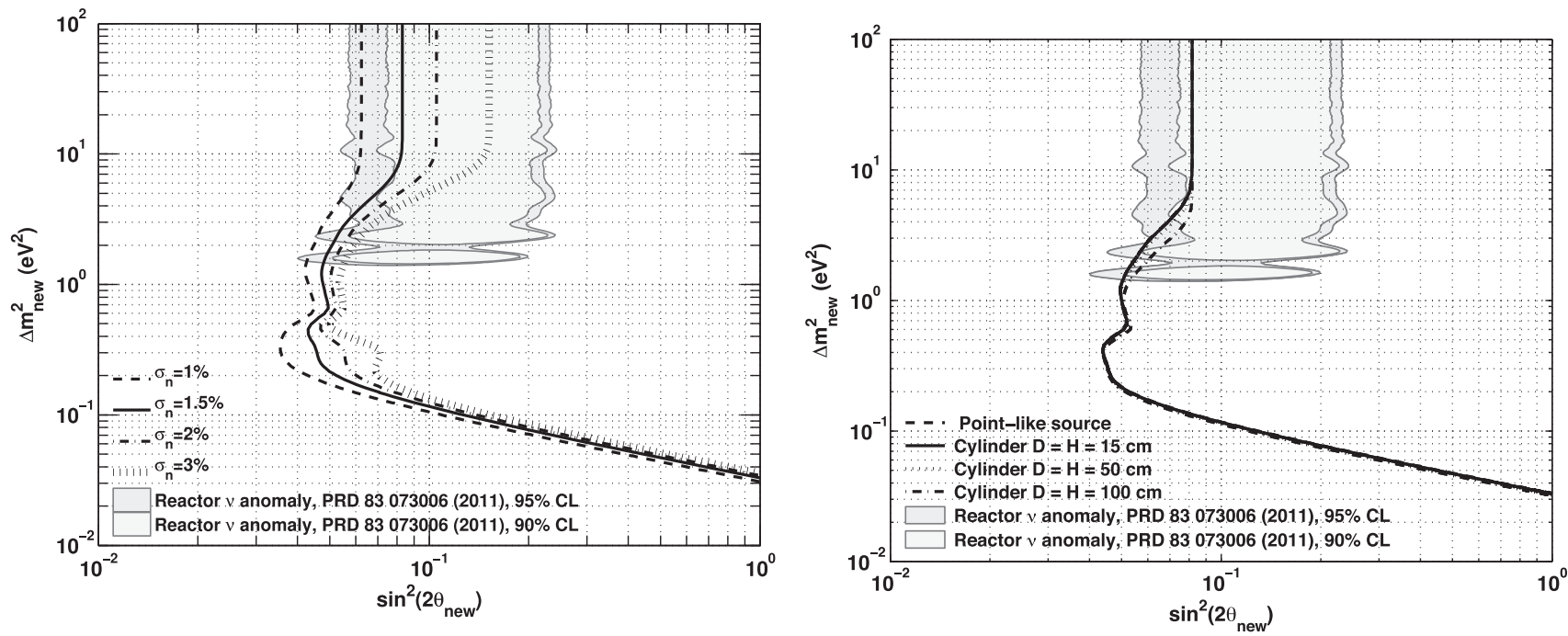

FIG. 11. Left panel: Influence of normalization uncertainty on sensitivity. Right panel: Influence of source extension on sensitivity. See Fig. 7 for other parameters. 
IBD events counting uncertainty and directly results in a better determination of the oscillation parameters. The $\chi^{2}$ contours are also impacted to a lesser extent in the intermediate $\Delta m_{\text {new }}^{2}$ region. Such a study demonstrates that measuring the source activity with a percent-level precision is therefore a prime requirement in order to ensure a good overall sensitivity.

\section{Source spatial extension}

Up to now, the $\mathrm{L}$ and $\mathrm{E}$ binned spectra modeling presented in Sec. VI A assumed the ${ }^{144} \mathrm{Ce}-{ }^{144} \mathrm{Pr}$ ANG to be a pointlike source. At the end of the manufacturing process, the ${ }^{144} \mathrm{Ce}_{-}{ }^{144} \mathrm{Pr}$ source will be packed in a cylindrical double capsule made of stainless steel, with equal diameter and height of roughly $15 \mathrm{~cm}$. This section investigates the effect of a spatially extended source on the sensitivity contours to short baseline oscillations.

For a spatially extended source, the modeling of the binned $\mathrm{L}$ and $\mathrm{E}$ spectra is done by averaging Eq. (1) on the source volume. This is done through a Monte Carlo simulation by randomly drawing many point sources in the cylindrical source volume and averaging the corresponding $\mathrm{L}$ and $\mathrm{E}$ spectra. The right panel of Fig. 11 shows the impact of the source spatial extension on the sensitivity contours, for cylinders of equal diameters and heights. Averaging Eq. (1) over the source volume makes the amplitude of the $\bar{\nu}_{e}$ oscillations damped with respect to Eq. (2), while keeping the same mean deficit. Therefore, sensitivity to short baseline oscillations is especially lost in the intermediate $\Delta m_{\text {new }}^{2}$ regime compared to the pointlike source case. Sensitivity remains almost unaffected in the low $\Delta m_{\text {new }}^{2}$ regime because, once again, the oscillation length is larger than the detector size and it makes the detector insensitive to any oscillation damping. In the high
$\Delta m_{\text {new }}^{2}$ region, contours are also unaffected by the source spatial extension. In this regime, the oscillation pattern is seriously damped by the detector vertex and energy resolution, making the sensitivity to short baseline oscillations entirely driven by a rate deficit.

As further shown by the right panel of Fig. 11, the spatial extension of a ${ }^{144} \mathrm{Ce}^{144} \mathrm{Pr}$ source with $H=D=15 \mathrm{~cm}$, such as the source provided by FSUE "Mayak" PA, has a negligible impact on the sensitivity contours with respect to the pointlike source case. Indeed, an oscillation half-length of $15 \mathrm{~cm}$ or lower corresponds to $\Delta m_{\text {new }}^{2}$ values above $20 \mathrm{eV}^{2}$ where the detector energy and distance resolutions have already damped the oscillation patterns. A source with spatial extension larger than the detector vertex resolution would be necessary to significantly degrade the sensitivity contours. A 1-m half oscillation length corresponds to $\Delta m_{\text {new }}^{2}$ around $3 \mathrm{eV}^{2}$ where logically the sensitivity is the most degraded with a hypothetical 1-m sized source.

\section{Source deployment location}

The three detectors suitable for a $\bar{\nu}_{e}$ source experiment at short baselines (see Sec. V) have quite a similar design. Any difference in the experimental configuration offered by such detectors then arises from the source deployment location with respect to the detector center. The deployment location directly determines the distance between the ANG and the detector center and therefore inversely quadratically scales the statistical uncertainties in the expected number of $\bar{\nu}_{e}$ event, both as a function of energy $E$ and baseline $L$. Therefore, the resulting effect on the sensitivity contours to short baseline oscillations is expected to be similar to the impact of the ANG activity.

Figure 12 shows the sensitivity contours for different source-to-detector distances. Both the rate + shape and free rate analysis are strongly impacted. The change in the
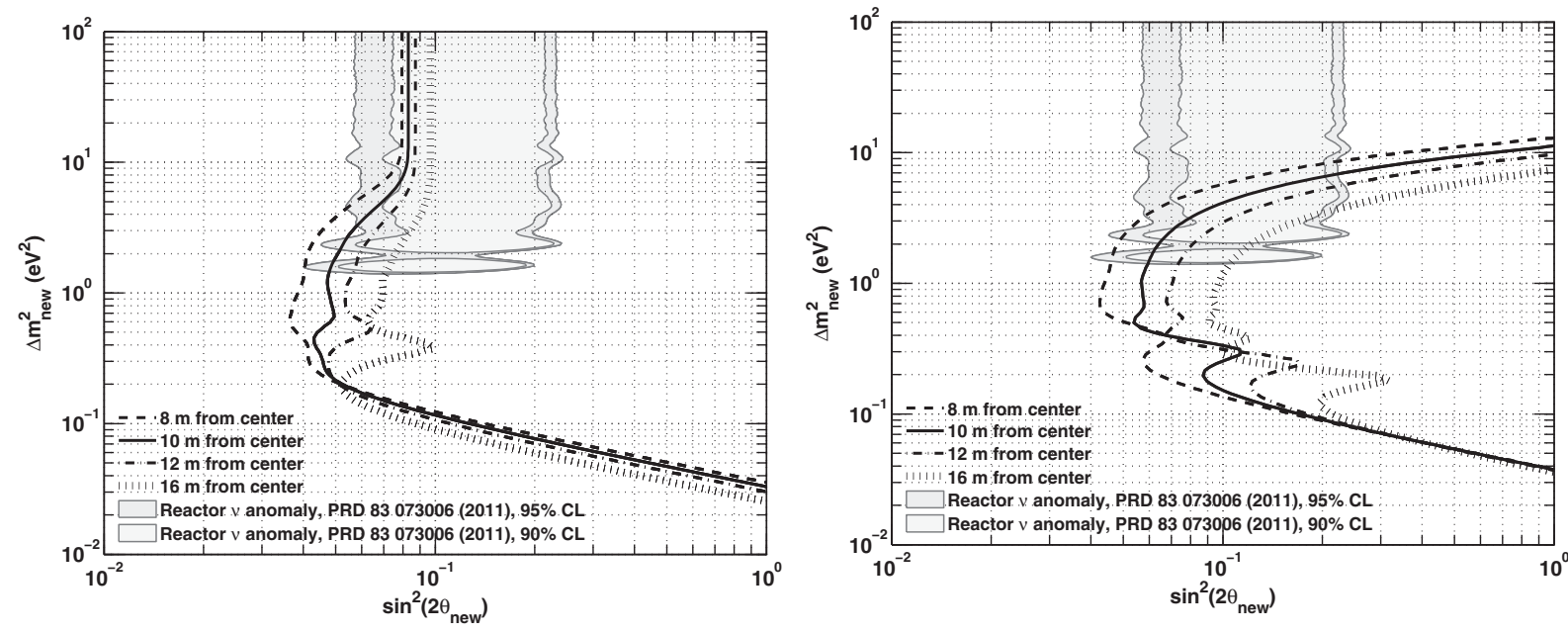

FIG. 12. Influence of distance between source and detector center on sensitivity, with a rate + shape analysis (left panel) and a free rate analysis (right panel). In the extreme case the overall statistics vary from $6.2 \times 10^{2}$ events to $2.7 \times 10^{4}$ events. See Fig. 7 for other parameters. 

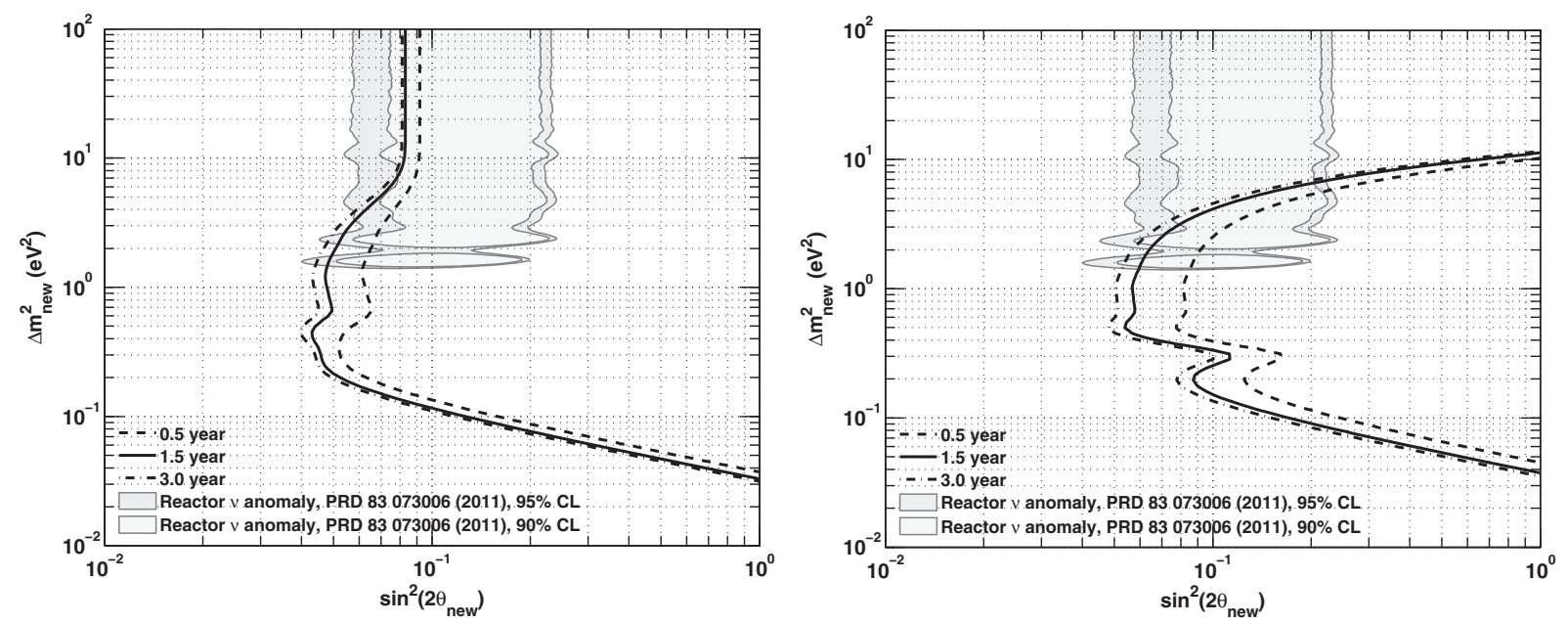

FIG. 13. Influence of data-taking time on sensitivity, with a rate + shape analysis (left panel) and a free rate analysis (right panel). See Fig. 7 for other parameters.

sensitivity contours at low and high $\Delta m_{\text {new }}^{2}$ is mostly due to the change in the statistical uncertainties caused by the source-detector distance because oscillations are unresolved in these regimes. However, the impact of the source-detector distance is quite different from the impact of the source activity in the $0.1 \mathrm{eV}^{2} \lesssim \Delta m_{\text {new }}^{2} \lesssim 0.5 \mathrm{eV}^{2}$ region. The "turnover point" between the low $\Delta m_{\text {new }}^{2}$ and intermediate $\Delta m_{\text {new }}^{2}$ regimes is reached when a first oscillation maximum starts to be fully contained and sampled by the detector. This situation depends both on the source location with respect to the detector center and the detector size. As shown by the right panel of Fig. 12, it corresponds to $\Delta m_{\text {new }}^{2}=0.2 \mathrm{eV}^{2}$ and $\sin ^{2}\left(2 \theta_{\text {new }}\right)=0.09$ for the generic experimental configuration described previously (i.e., an ANG located $10 \mathrm{~m}$ away from the center of a 5.5-m radius spherical detector). Detectors placed further away than $10 \mathrm{~m}$ from an ANG present "turnover points" in a free rate analysis which correspond to lower $\Delta m_{\text {new }}^{2}$ (i.e., larger oscillation periods) and lower $\sin ^{2}\left(2 \theta_{\text {new }}\right)$ values. This is, first, because of the resulting decrease in the statistical uncertainties and, second, because larger sourcedetector baselines enable detectors to be positioned on the maximum of oscillation patterns with larger periods.

From the event statistics point of view, the closer the ANG to the detector center the better. However, the smallest source-detector baseline might not be the optimal baseline if the source-induced gamma and neutron radiations can make a signicant level of background into the detector. This concern is especially important for the deployment of an ANG within a low background liquid scintillator detector such as those discussed in Sec. V.

The statistics is also enhanced by data-taking time. However, this effect is attenuated by the decrease in activity with time, as shown by Fig. 13. For our generic reference experiment, the expected number of events are $8.0 \times 10^{3}$, $1.6 \times 10^{4}$, and $2.1 \times 10^{5}$ for $0.5,1.5$, and 3 years of data taking, respectively, assuming a $100 \%$ detection efficiency. Therefore, the deployment of the ${ }^{144} \mathrm{Ce}^{144} \mathrm{Pr}$ ANG for 1.5 years is a good compromise, the sensitivity being marginally improved for longer data-taking times.

\section{Detector energy and vertex resolutions}

The effect of varying the detector energy and vertex resolutions on the sensitivity contours is shown on Figs. 14 and 15, respectively. The energy resolution of the generic detector considered in the present study was varied from $2.5 \%$ to $15 \%$ and was assumed to be independent of energy. The vertex resolution was varied from 5 to $50 \mathrm{~cm}$. The change in the sensitivity contours due to the degradation of energy and vertex resolution is similar, and occurs only in the $\Delta m_{\text {new }}^{2} \gtrsim 1 \mathrm{eV}^{2}$ region for a free rate analysis. As explained in Sec. VIA, and as shown in Fig. 6, the oscillation pattern is significantly washed out by the finite detector energy and vertex resolution for $\Delta m_{\text {new }}^{2} \gtrsim 1 \mathrm{eV}^{2}$. Concerning the rate + shape analysis, the sensitivity contours remain unchanged for $\Delta m_{\text {new }}^{2} \gtrsim 10 \mathrm{eV}^{2}$ because, in this region, the experiment is only sensitive to a rate deficit.

The three detectors discussed in Sec. V typically have energy resolutions and vertex resolutions of $7.5 \% / \sqrt{\mathrm{E}_{\mathrm{vis}}(\mathrm{MeV})}$ and $15 \mathrm{~cm} / \sqrt{\mathrm{E}_{\mathrm{vis}}(\mathrm{MeV})}$, respectively. As shown by Figs. 14 and 15, such detectors then present good enough vertex and energy reconstruction performances for a short baseline ANG experiment. However, calibration of the detector response to vertex and energy reconstruction has to be carefully done through the full detection volume, especially to pinpoint and correct for any position dependency.

\section{BACKGROUNDS}

As already stated in Sec. V, the backgrounds to $\bar{\nu}_{e}$ detection in the KamLAND, Borexino and $\mathrm{SNO}+$ 

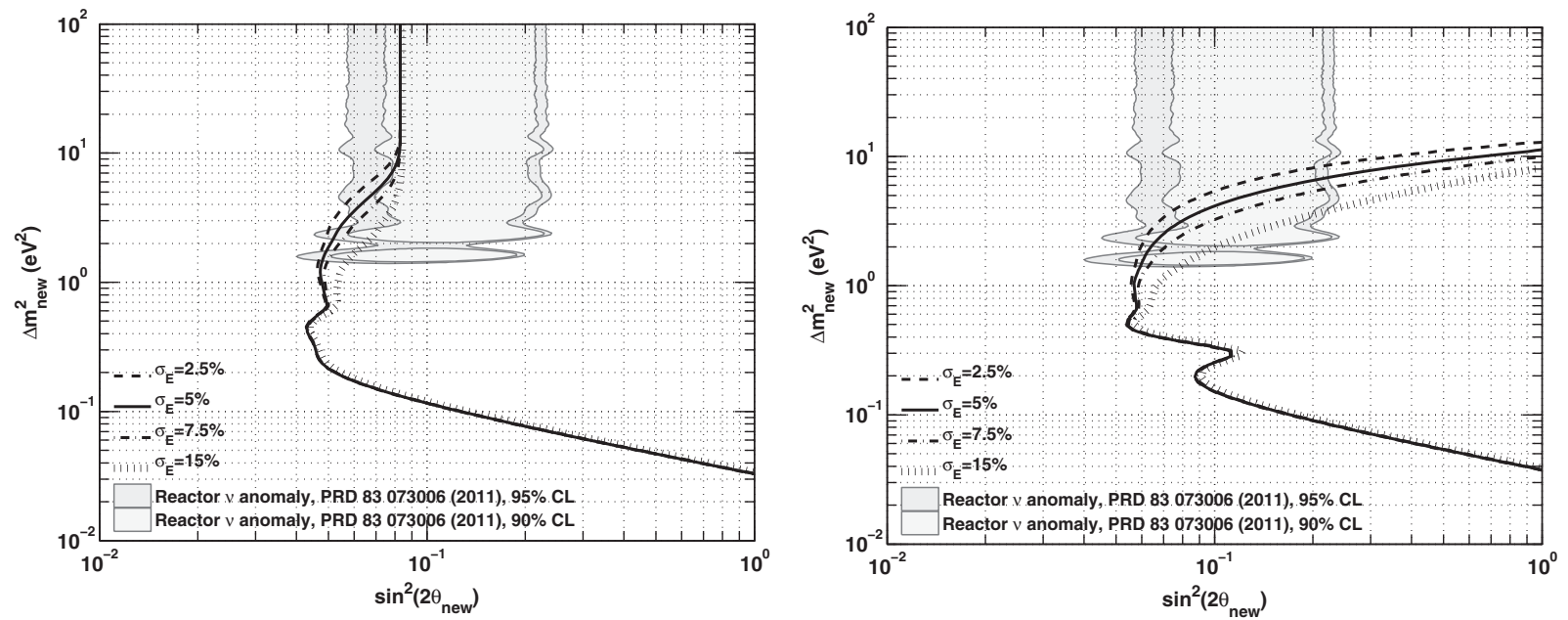

FIG. 14. Influence of energy resolution on sensitivity, with a rate + shape analysis (left panel) and a free rate analysis (right panel). See Fig. 7 for other parameters.

detectors are negligible, thanks to large overburdens and low radioactivity materials. However, radioactive contaminants left in the ANG after the manufacturing process can bring several backgrounds to the detector, divided into gamma and neutron contributions. This section discusses these possible source-induced backgrounds. A detailed simulation of the source-induced gamma and neutron backgrounds is presented and used to estimate the rate of backgrounds in the generic experimental configuration described previously (see Sec. VI) as a function of the level of radioactive contaminants in the ${ }^{144} \mathrm{Ce}-{ }^{144} \operatorname{Pr}$ source.

\section{A. TRIPOLI-4® simulation code}

Simulating the source-induced background is challenging, especially because the transportation of gammas and neutrons through media totaling an a $\gtrsim 10^{15}$ attenuation factor up to the detection volume is nearly impossible with brute-force Monte Carlo simulation (for example, standard

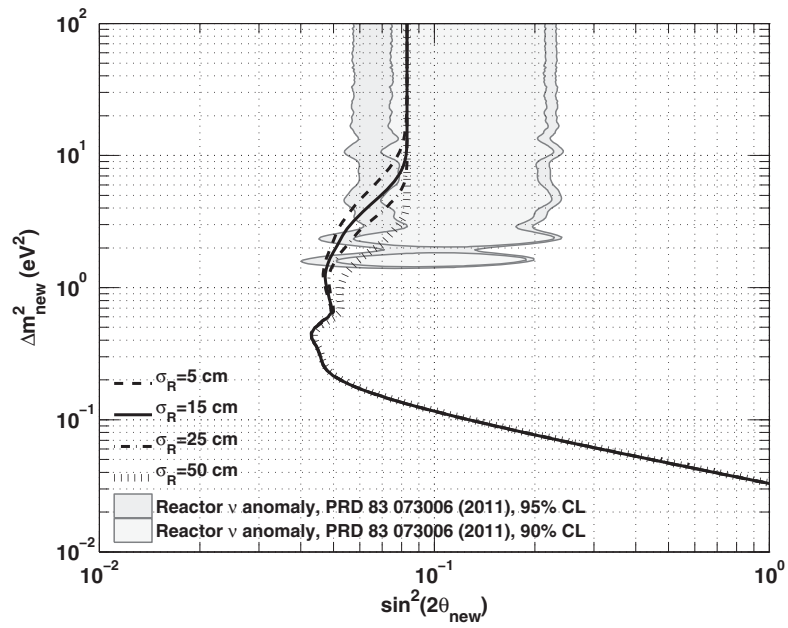

GEANT4). The TRIPOLI-4® Monte Carlo simulation code was then used [36]. This nuclear reactor physics code was designed to study criticality and to efficiently transport both neutrons and gammas with accurate models, based on databases of pointlike cross sections. It has been validated by numerous comparisons with experiment and is currently used by several French nuclear companies.

TRIPOLI-4® was chosen for two reasons: First, it offers state-of-the-art neutron transport modeling, and second, it incorporates different variance reduction techniques to deal with the high attenuation factors of both neutron and gamma transport in realistic configurations [36]. In particular, we used INIPOND, a special built-in module of TRIPOLI-4® based on the exponential transform method [37]. This exponential biasing is performed using an automatic precalculation of an importance map. The importance map provides information on the probability, for each point of the phase space, for a particle to reach the detector. It is

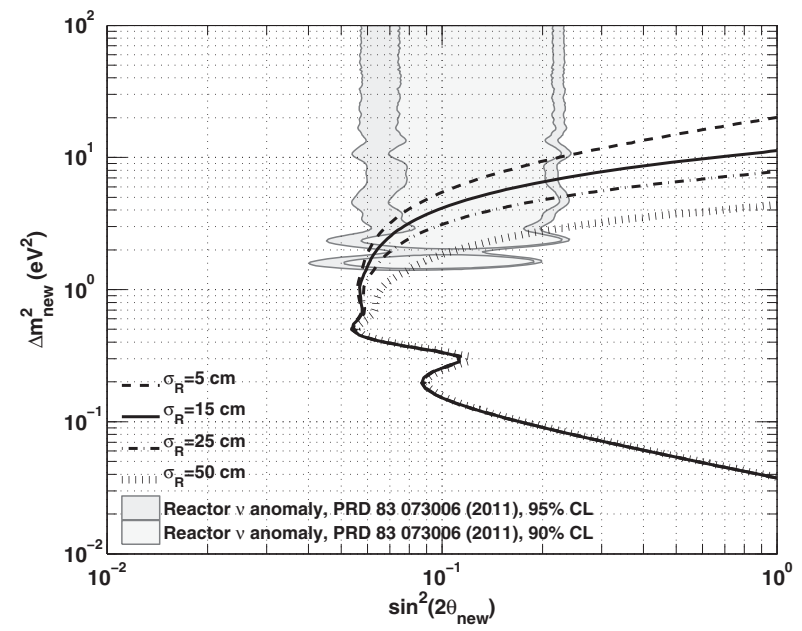

FIG. 15. Influence of vertex resolution on sensitivity, with a rate + shape analysis (left panel) and a free rate analysis (right panel). See Fig. 7 for other parameters. 
calculated with a simplified deterministic solver and can be used to calculate all possible particle paths over a virtual mesh of the geometry, together with their associated probabilities. It allows the code to adjust the weight of a particle traveling along a given path, in such a way that the mean quantities calculated by the code are unbiased while their variance is reduced. For example, below a given weight value, the particle can be killed (typically a particle moving downward in our generic reference experiment). This process allows a relatively large amount of particles to reach the target volume, such that it is possible to obtain statistically significant results. As shown in Sec. VII B, statistical uncertainty around $5 \%$ can be reached for attenuation as high as $10^{-18}$ with only $10^{9}$ events.

But on top of the statistical uncertainty, large systematic uncertainties must be considered. The attenuation is so important that any bias on cross section, density or attenuation coefficient can have a significant impact on the final results. Moreover, several parameters of the simulation have large uncertainties, such as the cerium oxide density in the source capsule. Attenuation coefficients and the different media density uncertainties result in an additional $10 \%$ to $20 \%$ systematic uncertainty. A conservative $50 \%$ systematic uncertainty is then affected to the simulation results presented in the next two sections. The quoted statistical uncertainties are an estimator of the convergence of the simulation.

Another drawback of the exponential biasing technique is that it prevents any track-by-track analysis, as can usually be done, for example, in GEANT4. Only predefined integral quantities, the so-called scores, are available, such as currents crossing a surface, fluxes, reaction rates and energy deposition estimators (for example, the energy of the incident particle if an energy deposition occurs). The true energy deposition is not available because the particle can survive an interaction with a lower weight, instead of releasing all its energy. The exponential biasing method has been compared to the natural simulation (also called analog transport) on a thinner geometry where the natural simulation could reach significant statistics. All simulation results were found to be in very good agreement.

\section{B. Source-induced gamma background}

Decay of ${ }^{144} \mathrm{Ce}$ and ${ }^{144} \mathrm{Pr}$ isotopes along with decay of radioactive impurities could be a source of gamma background for the experiment, especially if the source is close to the target volume. Such gamma backgrounds arise from the deexcitation of unstable daughter nuclei or bremsstrahlung of $\beta$ particles. The most serious source of gamma background is the $2.185-\mathrm{MeV}$ deexcitation gamma ray following the $\beta^{-}$decay of ${ }^{144} \mathrm{Pr}$, with an intensity of $0.7 \%$.

Still, other $\gamma$ rays and $\mathrm{x}$ rays can follow either from bremsstrahlung of $\beta$ particles or other deexcitation modes of ${ }^{144} \mathrm{Pr},{ }^{144} \mathrm{Ce}$, or lanthanide and actinide contaminants. Attenuation to $\gamma$ rays reaches a minimum of
$4.3 \times 10^{-2} \mathrm{~cm}^{2} / \mathrm{g}$ around $3.7 \mathrm{MeV}$ in tungsten, a value which is only $7 \%$ less than the $\gamma$ attenuation in tungsten at $2.185 \mathrm{MeV}$ [38]. Therefore, any $\gamma$ rays and $\mathrm{x}$ rays will be easily shielded as well. In other words, the ${ }^{144} \operatorname{Pr} 2.185$ $\mathrm{MeV}$ gamma emission drives the shielding thickness despite any realistic hypothesis on the source contamination by other radioisotopes, and the source-induced gamma background can be safely narrowed to the study of the ${ }^{144} \mathrm{Pr}$ $2.185 \mathrm{MeV}$ gamma ray.

The study of the ${ }^{144} \operatorname{Pr} 2.185-\mathrm{MeV}$ gamma-ray escape and transport to the generic experiment target volume is performed by generating monoenergetic gamma rays homogeneously and isotropically in the cerium oxide source. The use of the TRIPOLI-4® exponential biasing method discussed in Sec. VII A allowed us to reach significant results generating only $10^{9} \gamma$. The simulation shows statistical error bars around $2 \%$ per bin, as high as $5 \%$ for the 2.1-2.2-MeV bins. These statistical uncertainties indicate a reasonable convergence of the simulation.

Left panel of Fig. 16 shows the probability of a gamma to interact in the different media (steel plate, veto, buffer, target volume-see Fig. 5) along its path to the target volume as a function of energy. For instance, $6 \times 10^{-17}$ gamma per initial $2.185 \mathrm{MeV}$ gamma emitted by the source interact in the target with an incident energy in the range 1-2.4 MeV (the typical prompt energy window for the selection of IBD candidates). In the delayed energy window, only $9.3 \times 10^{-18}$ gamma per initial $2.185 \mathrm{MeV}$ gamma make an energy deposition in the target volume. The total current of the gamma ray with energies greater than $1 \mathrm{MeV}$ entering the target volume with an energy higher than $1 \mathrm{MeV}$ amounts to $6 \times 10^{-17} \gamma$ per initial $\gamma$, and is consistent with the previous numbers. Hence, the expected count rate of events above $1 \mathrm{MeV}$ from a sourceinduced gamma ray is $1.3 \times 10^{2}$ events/day for a $3.7 \mathrm{PBq}$ source. Therefore, assuming a 1-ms time coincidence window and no delayed energy cut, a rate of $2 \times 10^{-4}$ accidental IBD-like events per day is expected (1 event every 13 years). Applying a delayed energy cut at $>2 \mathrm{MeV}$ reduces the accidental rate to $3 \times 10^{-5}$ IBD-like events per day.

These results can be worthily compared to an analytical calculation of the attenuation $A$ of a gamma ray propagating along the $z$ axis from the source to the target:

$$
\mathrm{A}=\exp \left(-\sum_{k} e_{k} \lambda_{k} \rho_{k}\right),
$$

where $e_{k}, \rho_{k}$ and $\lambda_{k}$ are, respectively, the thickness, the density and the total attenuation coefficient of the $k$ th medium being crossed by the gamma ray from the source to the detection volume. An averaged material thickness must be considered for the $\mathrm{CeO}_{2}$ source material because gamma rays can be emitted anywhere along the height of the source. The $\mathrm{CeO}_{2}$ source material thickness is calculated according to Eq. (6): 

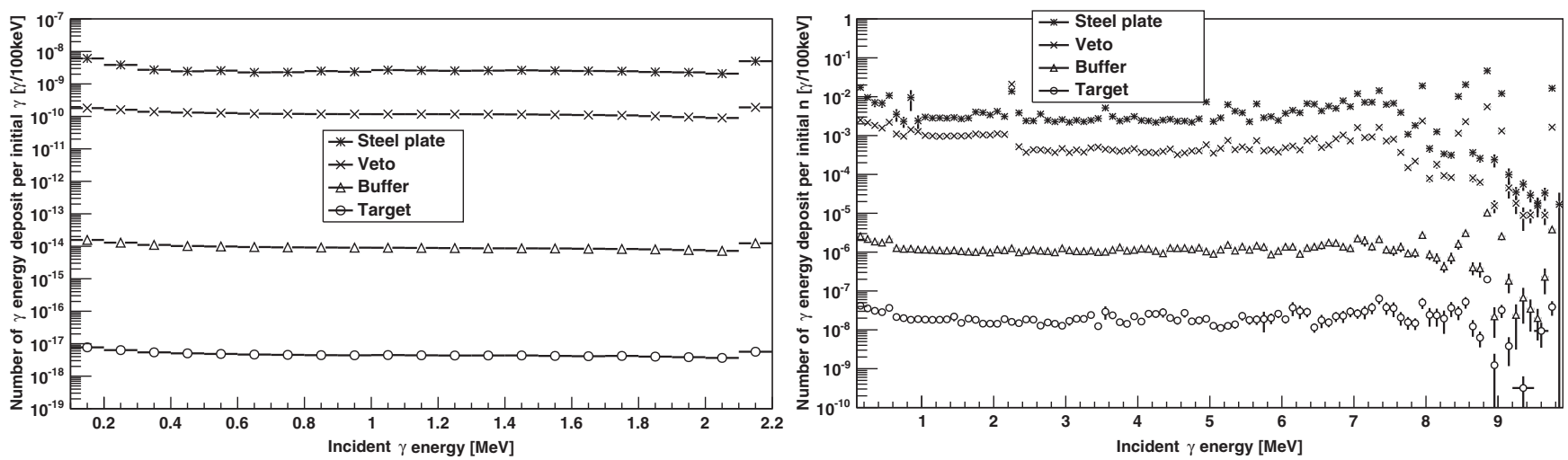

FIG. 16. Simulation of the number of gamma energy deposits from the steel plate to the target, per one initial gamma ray at $2.185 \mathrm{MeV}$ (left panel) and per one initial neutron shot in a Watt spectrum (right panel). Error bars are statistical only. Since the gamma simulation is much faster and easier to bias, the statistics is higher and error bars are contained in the points. The neutron simulation shows several points with very high error bars, a symptom of poor convergence in the considered bins. It is worth noting that direct neutron energy deposits are not considered here, only the energy deposits of gammas from neutron reactions. High-energy gammas mainly come from neutron capture on iron, as indicated by the peaks in the spectrum in the steel plate. At lower energy, the 2.2-MeV peak signs the neutron capture on hydrogen and the $0.5-\mathrm{MeV}$ peak comes from pair creation.

$$
\exp \left(-\rho \lambda e_{\mathrm{eff}}\right)=\frac{1}{H} \int_{0}^{H} e^{-x \rho \lambda} \mathrm{d} x
$$

with $H=14 \mathrm{~cm}$ the height of the source, which gives $e_{\text {eff }}=5.58 \mathrm{~cm}$.

Considering the media listed in Table IV along with their corresponding thickness, density and attenuation coefficient, the total attenuation factor is found to be $3.7 \times 10^{-16}$ at $2.185 \mathrm{MeV}$. The previous calculation must be refined by taking into account the source-detector solid angle. The solid angle can be calculated under the approximation of a pointlike source with Eq. (7):

$$
\frac{\Omega}{4 \pi}=\frac{1}{2}\left(1-\sqrt{1-\left(\frac{R}{L}\right)^{2}}\right),
$$

where $R$ is the radius of the detection volume and $L$ is the distance between the center of the source and the center of the target volume. For the generic reference experiment, $\Omega / 4 \pi=8.24 \%$, so that the total attenuation is $3 \times 10^{-17}$. This has to be compared with the $6 \times 10^{-18}$ attenuation

TABLE IV. Main materials contributing to gamma attenuation in our generic reference experiment. The total attenuation coefficient is taken from NIST's XCOM database [38]. The $\mathrm{CeO}_{2}$ thickness is the effective thickness as described in Eq. (6).

\begin{tabular}{lcccc}
\hline \hline Material & $\begin{array}{c}\text { Thickness } \\
(\mathrm{cm})\end{array}$ & $\begin{array}{c}\text { Density } \\
\left(\mathrm{g} / \mathrm{cm}^{3}\right)\end{array}$ & $\begin{array}{c}\text { Attenuation } \\
\text { coeff. }\left(\mathrm{cm}^{2} / \mathrm{g}\right)\end{array}$ & $\begin{array}{c}\text { Total } \\
\text { att. }\end{array}$ \\
\hline $\mathrm{CeO}_{2}$ & 5.58 & 4.5 & $4.06 \times 10^{-2}$ & $3.6 \times 10^{-1}$ \\
$\mathrm{~W}_{\text {alloy }}$ & 19 & 18.5 & $4.26 \times 10^{-2}$ & $3.1 \times 10^{-7}$ \\
Steel & 10 & 7.87 & $4.10 \times 10^{-2}$ & $4.0 \times 10^{-2}$ \\
Water & 200 & 1.0 & $4.69 \times 10^{-2}$ & $8.5 \times 10^{-5}$ \\
Oil & 200 & 0.77 & $4.50 \times 10^{-2}$ & $9.8 \times 10^{-4}$ \\
\hline \hline
\end{tabular}

factor in the 2.1-2.2-MeV energy range obtained from the simulation.

This calculation overestimates the number of $2.2-\mathrm{MeV}$ gamma rays reaching the target volume by a factor 5 . Actually, this calculation assumes that all gamma rays propagate along the $z$ axis and therefore with a minimal path length (i.e., $\mathrm{CeO}_{2}$ source thickness), while the simulation considers any paths from the source to the target volume.

Another refinement of the analytic modeling considers a coupled calculation of the solid angle and attenuation factor. The angle $\theta$ between the $z$ axis and the baseline defined by the source center and gamma interaction point is only considered here (i.e., the source is assumed to be spherical). As shown by Eq. (8), each material thickness is then increased by a factor $1 / \cos \theta$ :

$\mathrm{A}=\frac{1}{4 \pi} \int_{-\pi}^{\pi} \mathrm{d} \varphi \int_{0}^{\theta_{\max }} \exp \left(-\sum_{k} \frac{e_{k}}{\cos \theta} \lambda_{k} \rho_{k}\right) \sin \theta \mathrm{d} \theta$

where $\theta_{\max }$ is the maximum $\theta$ angle subtended by the detection volume and it is defined by $\sin \theta_{\max }=R / L$. Computing this equation numerically for the generic experiment configuration leads to an attenuation factor of $5 \times 10^{-18}$, similar to the simulation results. This simple model therefore brings useful information and allows us to correctly estimate the rate of source-induced gamma backgrounds in any experimental setup, without the need for a dedicated simulation package.

\section{Source-induced neutron background}

Actinide contaminants require much attention, since in addition to $\gamma$ and $\beta$ emission, they can undergo $\alpha$ decays and spontaneous fission (SF). Emission of $\beta$ and $\gamma$ rays will 
be completely suppressed by the tungsten shielding, as explained in the previous section, whereas $\alpha$ particle emission will be absorbed by the source itself. Actinides emit fast neutrons through $\alpha$ interaction with light nuclei such as oxygen in the $\mathrm{CeO}_{2}$ or spontaneous fission. The latter process is particularly dominant for neutron-rich heavy nuclei with an even number of nucleons. Those fast neutrons can easily escape the source despite the high- $Z$ shielding and scatter out in the detector materials to finally produce gamma rays outside the shielding through radiative capture. Such captures mostly occur on hydrogen atoms in water and oils (such as nondoped scintillators), producing 2.2-MeV $\gamma$ rays which can mimic both the prompt and the delayed events of an IBD reaction. As opposed to the $2.185-\mathrm{MeV} \gamma$ rays that can be attenuated through the high$Z$ shielding, the neutron emission could be the dominant source of induced background events even though the actinide contamination level is small.

However, the specific neutron activity of each of the actinide contaminants has to be balanced by their respective abundance in spent nuclear fuel. Fresh nuclear fuel does not contain any isotope heavier than ${ }^{238} \mathrm{U}$. All heavy isotopes are produced through a long chain of neutron capture and $\beta^{-}$decay. Moreover, nuclei with an odd number of nucleons generally have large fission cross sections, counterbalancing the radiative capture chain and the production of heavier isotopes. A burn-up threshold is therefore expected, depending on the isotope mass, together with a constant ratio between heavy isotope quantities, depending on cross sections, neutron fluxes and decays. Therefore, minor actinides are produced much less than lanthanides during irradiation. Furthermore, the heavier the actinide, the lower its abundance in spent nuclear fuel. This effect will compensate the increase of the branching ratio to SF with the number of nucleons.

Table V shows the half-life, branching ratio to SF, and specific neutron activity for the most produced isotopes of americium and curium with half-life higher than 180 days. It also provides a relative estimate of the activity of these minor actinides in 2-year-old spent nuclear fuel, normalized to the ${ }^{144} \mathrm{Ce}$ activity. The nuclei of interest are the even curium isotopes $\left({ }^{244,246,248} \mathrm{Cm}\right)$ because all americium isotopes and odd nucleon number isotopes have a very low branching ratio to SF $\left(\lesssim 10^{-5} \%\right)$. Berkelium isotopes have a very short period, and nuclei heavier than ${ }^{248} \mathrm{Cm}$ $\left({ }^{250} \mathrm{Cm}, \mathrm{Cf}, \mathrm{Es}, \mathrm{Fm}\right)$ can be safely ignored because either their abundance in spent nuclear fuel is negligible or their half-life period is short. It is worth noting that the mean number of neutrons released per SF $v$ increases with the nucleus mass, following roughly $v=0.1094 A-23.94$ in the mass range of the previously discussed actinide contaminants, so that ${ }^{248} \mathrm{Cm}$ releases $33 \%$ more neutrons per SF than ${ }^{241} \mathrm{Am}(v=3.2$ and $v=2.4$, respectively).

Taking into account all these effects shows that the ${ }^{244} \mathrm{Cm}$ is the most problematic nucleus from the SF-induced
TABLE V. Neutron emission and activity $\mathcal{A}$ relative to ${ }^{144} \mathrm{Ce}$ activity of the most produced $\mathrm{Am}$ and $\mathrm{Cm}$ isotopes (National Nuclear Data Center, information extracted from the Sigma database [39]).

\begin{tabular}{|c|c|c|c|c|}
\hline Isotope & Half-life & $I_{\mathrm{SF}}(\%)$ & $\begin{array}{c}\text { Specific } \\
\text { neutron } \\
\text { activity }(\mathrm{n} / \mathrm{g})\end{array}$ & $\begin{array}{l}\mathcal{A} / \mathcal{A}\left({ }^{144} \mathrm{Ce}\right) \text { in } \\
\operatorname{SNF}(\mathrm{Bq} / \mathrm{Bq})\end{array}$ \\
\hline${ }^{241} \mathrm{Am}$ & $432.2 \mathrm{y}$ & $4.0 \times 10^{-10}$ & 1.2 & $5 \times 10^{-03}$ \\
\hline${ }^{242 m} A m$ & $141 \mathrm{y}$ & $4.7 \times 10^{-9}$ & 46 & $1 \times 10^{-04}$ \\
\hline${ }^{243} \mathrm{Am}$ & $7370 \mathrm{y}$ & $3.7 \times 10^{-9}$ & 0.72 & $2 \times 10^{-04}$ \\
\hline${ }^{240} \mathrm{Cm}$ & $27 \mathrm{~d}$ & $3.9 \times 10^{-6}$ & $6.7 \times 10^{7}$ & \\
\hline${ }^{242} \mathrm{Cm}$ & $162.8 \mathrm{~d}$ & $6.2 \times 10^{-6}$ & $1.9 \times 10^{7}$ & \\
\hline${ }^{243} \mathrm{Cm}$ & $29.1 \mathrm{y}$ & $5.3 \times 10^{-9}$ & $2.6 \times 10^{2}$ & $2 \times 10^{-04}$ \\
\hline${ }^{244} \mathrm{Cm}$ & $18.10 \mathrm{y}$ & $1.4 \times 10^{-4}$ & $1.6 \times 10^{7}$ & $2 \times 10^{-02}$ \\
\hline${ }^{245} \mathrm{Cm}$ & $8.5 \times 10^{3} y$ & $6.1 \times 10^{-7}$ & $1.1 \times 10^{2}$ & $3 \times 10^{-06}$ \\
\hline${ }^{246} \mathrm{Cm}$ & $4.73 \times 10^{3} \mathrm{y}$ & $3.0 \times 10^{-2}$ & $1.0 \times 10^{7}$ & $5 \times 10^{-07}$ \\
\hline${ }^{248} \mathrm{Cm}$ & $3.40 \times 10^{5} \mathrm{y}$ & 8.39 & $4.2 \times 10^{7}$ & $<1 \times 10^{-07}$ \\
\hline
\end{tabular}

background point of view, with a 18-year lifetime, a branching ratio to $\mathrm{SF}$ of $1.4 \times 10^{-4} \%$ and a typical production of a few grams per fuel assembly in standard VVER-400 cycles [40,41]. Roughly speaking, the expected production of isotopes heavier than ${ }^{244} \mathrm{Cm}$ is lower by 1 order of magnitude per additional nucleon, leading to negligible quantities of $\mathrm{Cf}$ and heavy $\mathrm{Cm}$ isotopes. All together, minor actinides (therefore excluding $\mathrm{U}, \mathrm{Pu}$ and $\mathrm{Np}$ ) will produce about $10^{7}$ neutrons/g after 3 years of cooling, for typical VVER-400 spent fuel [42]. The actinides are efficiently separated from cerium during the ANG production, but remaining trace contaminations are expected. Assuming $10^{-5} \mathrm{~Bq}$ of ${ }^{244} \mathrm{Cm}$ per Bq of ${ }^{144} \mathrm{Ce}$, we computed that $1.4 \times 10^{5} \mathrm{n} / \mathrm{s}$ are expected to be emitted by the ANG.

The simulation of source-induced neutron background in the generic reference experiment is done by randomly shooting single neutrons following a Watt fission spectrum, and distributed homogeneously in the source. Only $60 \%$ of neutrons are captured by the shielding. Surviving neutrons are captured in the ground, in the steel plate or in the veto.

TABLE VI. Summary of the expected $\bar{\nu}_{e}$ daily signal and ANGinduced background rates for the generic experimental configuration. A contamination of $10^{-5} \mathrm{~Bq}$ of ${ }^{244} \mathrm{Cm}$ per $\mathrm{Bq}$ of ${ }^{144} \mathrm{Ce}$ is assumed. IBD-like events are selected as follows: $\mathrm{E}_{\text {prompt }}$ in 1-2.4 MeV, $\mathrm{E}_{\text {delayed }}$ in $2.0-2.4 \mathrm{MeV}$ and $\Delta t_{p d}<1 \mathrm{~ms}$. Backgrounds are varying quadratically with the activity.

\begin{tabular}{lccc}
\hline \hline $\begin{array}{c}\bar{\nu}_{e} \\
\text { Activity }\end{array}$ & $\begin{array}{c}\gamma \text { induced } \\
\text { IBD-like signal }\end{array}$ & $\begin{array}{c}\text { Neutron induced } \\
\text { IBD-like signal }\end{array}$ \\
\hline $5.5 \mathrm{PBq}$ & 80 & $6.5 \times 10^{-5}$ & $3.6 \times 10^{-2}$ \\
$3.7 \mathrm{PBq}$ & 54 & $3.0 \times 10^{-5}$ & $1.6 \times 10^{-2}$ \\
$2.7 \mathrm{PBq}$ & 39 & $1.5 \times 10^{-5}$ & $0.1 \times 10^{-2}$ \\
$1.7 \mathrm{PBq}$ & 25 & $0.6 \times 10^{-5}$ & $0.3 \times 10^{-2}$ \\
$0.9 \mathrm{PBq}$ & 13 & $0.2 \times 10^{-5}$ & $0.1 \times 10^{-2}$ \\
\hline \hline
\end{tabular}


Captures on metal produce high-energy gamma rays (6-9 MeV), while captures on hydrogen in the veto produce 2.2-MeV gamma rays. All of these gamma rays are then degraded on their travel to the target volume, which tends to flatten the capture peaks.

The right panel of Fig. 16 shows the probability of making a gamma energy deposition in the different detector subvolumes, consequently, to the emission of one neutron, as obtained with the TRIPOLI-4® software. For instance, the probability of making a gamma energy deposition in the $1-2.4-\mathrm{MeV}$ prompt energy range is $2.4 \times 10^{-7}$, with an almost flat spectrum in this range. In the $2.0-2.4-\mathrm{MeV}$ delayed energy range, the corresponding probability is $0.6 \times 10^{-7}$. A contamination of $10^{-5} \mathrm{~Bq}$ of ${ }^{244} \mathrm{Cm}$ per $\mathrm{Bq}$ of ${ }^{144} \mathrm{Ce}$ would lead to 0.1 and $\sim 0.02$ accidental IBD-like events per day without and with a $>2 \mathrm{MeV}$ energy cut for the delayed event selection, respectively. The neutroninduced accidental background would then still be negligible as compared to the expected $\bar{\nu}_{e}$ rate, although it is higher than the gamma-induced accidental background by 3 orders of magnitude.

Fast neutron emission from actinide contaminants in the source could also, in principle, be a source of correlated backgrounds. The first type of correlated background arises from single fast neutrons reaching the detection volume. Such neutrons can make a proton recoil and fake a prompt signal. Then, the neutron capture would complete the coincidence signal with a correlation time similar to IBD, the latter being driven by neutron thermalization and diffusion. However, neutrons have a very small probability to cross the inactive regions of the detector. Despite the use of the exponential biasing techniques in TRIPOLI-4®, no neutrons reached the detector target in our simulations, and only a few of them reached the buffer, leading to an upper limit of $1 \times 10^{-12}$ neutrons reaching the buffer per initial neutron. Furthermore, only the most energetic neutrons reaching the liquid scintillator volume would be able to make an energy deposition larger than the IBD 1-MeV visible energy threshold. The probability of such an energy deposition to be observed is even further reduced because of the high quenching factor of protons in liquid scintillators.

The second type of potential correlated background follows from multiple neutron emission in actinide SF. Two neutrons could scatter out of the source in the detector direction and be captured on hydrogen inside or close to the detection volume. The $\gamma$ rays from both captures would finally fake the IBD signal, the time correlation being ensured by the capture time of the two neutrons. Since two neutron-induced energy depositions are required to mimic an IBD signal, this background scales quadratically with the energy deposition in the scintillator per initial neutron. The TRIPOLI-4® simulation of neutrons for the generic reference experiment was unable to reproduce such correlated events in the target volume. Interpolating the results obtained from a simulation of an experimental configuration with thinner veto and buffer volumes, an upper limit of $10^{-4}$ IBD-like events per day could be derived. Finally, the combination of a ${ }^{244} \mathrm{Cm}$ contamination of less than $\mathcal{O}\left(10^{-4} \mathrm{~Bq} / \mathrm{Bq}\right)$ combined with the thick veto and buffer detector volumes would lead to negligible source-induced correlated backgrounds.

In any deployment scenario, neutron background could be further reduced by adding a neutron shielding, made of a moderator and a neutron absorber. Typical neutron moderators are neutron-rich materials, such as mineral oils, water or plastics. Using boron is the common solution to absorb neutrons without gamma emission because the ${ }^{10} \mathrm{~B}(n, \alpha)$ reaction has a very high cross section and releases only a 478-keV gamma ray. Outside the detector, the ANG would typically be shielded with borated polyethylene or polyethylene coated by boron carbide $\mathrm{B}_{4} \mathrm{C}$. With an ANG at the center of a detector, neutron backgrounds (if any) would tremendously complicate or even prevent a source experiment such as that described here. A dedicated neutron shielding surrounding the gamma shielding would be mandatory, such as a balloon filled with saturated boric water. It is worth noting that even with such a configuration, about $1 / 10^{4}$ neutrons are captured on hydrogen since the ratio of thermal radiative capture cross sections is [39]

$$
\frac{\sigma^{{ }^{10} \mathrm{~B}(n, \alpha)}}{\sigma_{\mathrm{H}(n, \gamma)}}=\frac{3803 \mathrm{~b}}{0,3284 \mathrm{~b}}=1.16 \times 10^{4} .
$$

This ratio could be increased by a factor 656 using heavy water since the deuterium thermal radiative capture cross section is only $5.0 \times 10^{-4} \mathrm{~b}$ [39].

\section{CONCLUSION}

A definitive test of the short baseline anomalies is necessary to address the hypothesis of a light sterile neutrino. A smoking-gun signature of neutrino oscillations at short distances is the observation of an oscillation pattern both in the reconstructed energy spectrum and spatial distribution of the IBD events. Such an observation can be performed in the short term and at a (relatively) modest cost through the deployment of an intense ANG near an existing large liquid scintillator detector. The ${ }^{144} \mathrm{Ce}^{-144} \mathrm{Pr}$ couple has been identified to be the most suitable $\bar{\nu}_{e}$ emitter for this type of experiment. The production of a ${ }^{144} \mathrm{Ce}-{ }^{144} \mathrm{Pr}$ ANG with some PBq activity is technically feasible and can be realized by the Russian FSUE "Mayak" PA company by reprocessing spent nuclear fuel. Detectors with ultralow background levels and good vertex and energy reconstruction capabilities are necessary for the observation of an oscillation pattern in the $\left(\Delta m_{\text {new }}^{2}, \sin ^{2}\left(2 \theta_{\text {new }}\right)\right)$ parameter space relevant to the neutrino anomalies at short baselines. Three large liquid scintillator detectors which meet these requirements have been identified and are, namely, the 
KamLAND, Borexino and SNO+ detectors. Deploying an intense ANG next to one of these detectors allows us to completely neglect any kind of detector-induced backgrounds. As pointed out in this article, another possible source of backgrounds comes from the ANG itself. It has been shown that the impact of the source-induced backgrounds can be severely limited provided that the ANG is enclosed in a thick $\sim 20$-cm high-density material shielding (such as tungsten) and that the neutron emitting radioactive contaminants, which are leftovers resulting from the source manufacturing process, are kept at sufficiently low levels.

More precisely, the activity of any $\gamma$ emitters in the source must be smaller than $1 \%$ relative to the ANG absolute activity, such that the thickness of the high-density material shielding is driven by the activity of the 2.185$\mathrm{MeV}$ deexcitation gamma ray following the $\beta^{-}$decay of ${ }^{144} \mathrm{Pr}$. The contamination of minor actinides must be less than $10^{-5} \mathrm{~Bq}$ of actinide per $\mathrm{Bq}$ of ${ }^{144} \mathrm{Ce}$. Such a contamination level brings the rate of the neutron-induced accidental background to the rate of the $\gamma$-induced accidental background. A detailed simulation of gamma and neutron particle transport from the source up to the detection volume confirmed that the resulting backgrounds are negligible. Even though slightly higher contamination levels were observed, the sensitivity and physics reach of the experiment would not be degraded.

The ANG absolute activity is also another important parameter to control, especially for optimizing the experiment sensitivity in the high $\Delta m_{\text {new }}^{2} \gtrsim 10 \mathrm{eV}^{2}$. As such, the amount of $\beta$ radioactive impurities in the source must then be kept at a level of $0.1 \%$ or less to prevent any bias in the determination of the ANG activity with a calorimetric method. Moreover, the source $\beta$ and $\bar{\nu}_{e}$ spectrum shapes must be precisely measured in order not to spoil both the precision of the ANG absolute activity and the experiment sensitivity in a rate free analysis.

Having these source and detector requirements fulfilled ensures the sensitivity of such an experimental configuration to be mostly driven by statistical uncertainties. The best experimental scenario then corresponds to the biggest detector, the closest deployment location and the highest source activity. If any, higher unexpected source-induced backgrounds could be mitigated by a more distant deployment location and additional high- and/or lowdensity material shieldings.

To assess the impact of a future ANG experiment for ruling out the electron disappearance anomalies, we combined the reactor and gallium results with the future measurements of each ANG experiment. We used the parameter goodness of fit (PGof) test based on the difference between the overall best fit $\chi^{2}$ and the sum of the best fit $\chi^{2}$ 's for each ANG experiment only and the combination of both the reactor and gallium anomalies [43]. If any ANG experiment reported a no-oscillation result, the PGof resulting probabilities would be down to the level of $1 \%-1.5 \%$. The allowed mixing angles would be restricted to $\sin ^{2}\left(2 \theta_{\text {new }}\right) \lesssim 0.05$ for $\Delta m_{\text {new }}^{2} \lesssim 1.5 \mathrm{eV}^{2}$.

However, the sensitivity of the ANG experiment could be improved by either increasing the source activity (if technologically feasible) or by deploying the source closer to the active target volume or even inside the detector. The latter cases would necessitate a refurbishment of the detector as well as a more demanding control of the sourceinduced backgrounds. In particular, the mitigation of the neutron-induced backgrounds would need stringent requirements on the minor actinide contaminants, probably leading to further purification steps during production.

If there is a hint of any oscillation signal, another option would be to relocate the ANG at a slightly different baseline during the course of the experiment. The comparison of data at two different distances would be a reliable test against any oscillation scenario.

\section{ACKNOWLEDGMENTS}

We thank the KamLAND Collaboration for their active work and full support towards the investigation of the CeLAND experimental configuration. We acknowledge the continuous strong support of the Borexino Collaboration for the realization of the CeSOX experiment at the Laboratori Nazionali del Gran Sasso. We are grateful to the Russian FSUE "Mayak" PA company for their research and developments towards the realization of an intense ${ }^{144} \mathrm{Ce}$ ANG. Finally, we would like to thank V. Kornoukhov for continuous efforts on the engineering of the ${ }^{144} \mathrm{Ce}$ ANG. T. L. thanks the European Research Council for support under the Starting Grant No. StG-307184.
[1] J. Beringer et al. (Particle Data Group), Phys. Rev. D 86, 010001 (2012).

[2] A. Aguilar-Arevalo et al. (LSND Collaboration), Phys. Rev. D 64, 112007 (2001).

[3] A. Aguilar-Arevalo et al. (MiniBooNE Collaboration), Phys. Rev. Lett. 102, 101802 (2009).
[4] A. Aguilar-Arevalo et al. (MiniBooNE Collaboration), Phys. Rev. Lett. 105, 181801 (2010).

[5] C. Giunti and M. Laveder, Mod. Phys. Lett. A 22, 2499 (2007).

[6] C. Giunti and M. Laveder, Phys. Rev. C 83, 065504 (2011). 
[7] C. Giunti, M. Laveder, Y. Li, Q. Liu, and H. Long, Phys. Rev. D 86, 113014 (2012).

[8] G. Mention, M. Fechner, Th. Lasserre, Th. A. Mueller, D. Lhuillier, M. Cribier, and A. Letourneau, Phys. Rev. D 83, 073006 (2011).

[9] K. Abazajian et al., Light Sterile Neutrinos: A White Paper (Virginia Tech, 2012).

[10] A. Ianni, D. Montanino, and G. Scioscia, Eur. Phys. J. C 8, 609 (1999).

[11] M. Cribier, M. Fechner, T. Lasserre, A. Letourneau, D. Lhuillier, G. Mention, D. Franco, V. Kornoukhov, and S. Schönert, Phys. Rev. Lett. 107, 201801 (2011).

[12] K. Eguchi et al. (KamLAND Collaboration), Phys. Rev. Lett. 90, 021802 (2003).

[13] G. Alimonti et al. (Borexino Collaboration), Nucl. Instrum. Methods Phys. Res., Sect. A 600, 568 (2009).

[14] M. C. Chen (SNO+ Collaboration), arXiv:0810.3694.

[15] M. Cribier, L. Gosset, P. Lamare, J. Languillat, P. Perrin et al., Nucl. Instrum. Methods Phys. Res., Sect. A 378, 233 (1996).

[16] J. Abdurashitov et al. (SAGE Collaboration), Phys. Rev. C 59, 2246 (1999).

[17] J. Abdurashitov, V. Gavrin, S. Girin, V. Gorbachev, P. Gurkina et al., Phys. Rev. C 73, 045805 (2006).

[18] National Nuclear Data Center, information extracted from the Chart of Nuclides database, http://www.nndc.bnl.gov/ chart/.

[19] V. Gelis and G. Maslova, Radiokhimiya 40, 52 (1998); [Radiochemistry 40, 55 (1998)].

[20] V. Gelis, G. Maslova, and E. Chuveleva, Radiokhimiya 40, 55 (1998); [Radiochemistry 40, 59 (1998)].

[21] ${ }^{144}$ Ce specific activity is $118 \mathrm{TBq} / \mathrm{g}$ [22]. Depending on the spent fuel cooling time, the ${ }^{144} \mathrm{Ce}$ mass fraction among all stable isotopes is $0.1 \%-1 \%$.

[22] A. Gerasimov, V. Kornoukhov, I. Sal'dikov, and G. Tikhomirov, At. Energ. 116, 54 (2014).

[23] National Nuclear Data Center, "ENSDF Analysis Programs-LOGFT," http://www.nndc.bnl.gov/nndcscr/ensdf _pgm/analysis/logft.

[24] T. A. Mueller, D. Lhuillier, M. Fallot, A. Letourneau, S. Cormon, M. Fechner, L. Giot, T. Lasserre, J. Martino,
G. Mention, A. Porta, and F. Yermia, Phys. Rev. C 83, 054615 (2011).

[25] P. Huber, Phys. Rev. C 84, 024617 (2011).

[26] D. Wilkinson, Nucl. Instrum. Methods Phys. Res., Sect. A 290, 509 (1990).

[27] A. Sirlin, Phys. Rev. D 84, 014021 (2011).

[28] H. Behrens and W. Bühring, Electron Radial Wave Functions and Nuclear Beta-Decay, The International Series of Monographs on Physics Series (Clarendon Press, Oxford University Press, Great Clarendon Street, Oxford, OX2 6DP, 1982).

[29] P. Huber, Phys. Rev. C 85, 029901 (2012).

[30] F. Calaprice (private communication).

[31] A. Hayes, J. Friar, G. Garvey, G. Jungman, and G. Jonkmans, Phys. Rev. Lett. 112, 202501 (2014).

[32] G. Knoll, Radiation Detection and Measurement, 3rd ed. (John Wiley \& Sons, Hoboken, NJ, 2000).

[33] The water Cerenkov light yield is about 300 photons/MeV, compared to roughly $110^{4}$ photons $/ \mathrm{MeV}$ for a typical liquid scintillator.

[34] A. Gando, Y. Gando, S. Hayashida, H. Ikeda, K. Inoue et al., arXiv:1312.0896.

[35] D. D'Angelo et al. (Borexino Collaboration), arXiv: 1405.7919 .

[36] E. Brun et al., Ann. Nucl. Energy (to be published).

[37] F. Clark, The Exponential Transform as an Importance Sampling Device, A Review, Technical Report No. ORNL-RSIC-14 (Oak Ridge National Laboratory, Oak Ridge, TN, 1966).

[38] M. Berger, J. Hubbell, S. Seltzer, J. Chang, J. Coursey, R. Sukumar, D. Zucker, and K. Olsen, Xcom: Photon Cross Sections Database, National Institute of Standards and Technology, http://www.nist.gov/pml/data/xcom/index.cfm.

[39] National Nuclear Data Center, information extracted from the Sigma database, http://www.nndc.bnl.gov/sigma/.

[40] V. Kornoukhov, Report No. ITEP-2-97, 1997.

[41] T. Zaritskaya and A. Rudik, Report No. ITEP-101, 1981.

[42] V. N. Kornoukhov (private communication).

[43] M. Maltoni and T. Schwetz, Phys. Rev. D 68, 033020 (2003). 